\title{
ON THE HYPERBOLIC RELAXATION OF THE CAHN-HILLIARD EQUATION IN 3-D: APPROXIMATION AND LONG TIME BEHAVIOUR
}

\author{
ANTONIO SEGATTI
}

\begin{abstract}
In this paper we consider the hyperbolic relaxation of the Cahn-Hilliard equation ruling the evolution of the relative concentration $u$ of one component of a binary alloy system located in a bounded and regular domain $\Omega$ of $\mathbb{R}^{3}$. This equation is characterized by the presence of the additional inertial term $\varepsilon u_{t t}$ that accounts for the relaxation of the diffusion flux. For this equation we address the problem of the long time stability from the point of view of global attractors. The main difficulty in dealing with this system is the low regularity of its weak solutions, which prevents us from proving a uniqueness result and a proper energy identity for the solutions. We overcome this difficulty by using a density argument based on a Faedo-Galerkin approximation scheme and the recent J.M. Ball's theory of generalized semiflows. Moreover, we address the problem of the approximation of the attractor of the continuous problem with the one of Faedo-Galerkin scheme. Finally, we show that the same type of results hold also for the damped semilinear wave equation when the nonlinearity $\phi$ is not lipschitz continuous and has a super critical growth.
\end{abstract}

\section{Contents}

1. Introduction

2. Mathematical setting and weak formulation 5

3. Generalized semiflows 6

3.1. A weaker notion of attractor. 9

4. Faedo Galerkin approximation of the hyperbolic relaxation of the Cahn-Hilliard equation

5. Long time behaviour of the hyperbolic relaxation of the Cahn-Hilliard equation: existence of the global attractor

6. Approximation of the weak global attractor

7. Weak global attractor for the damped semilinear wave equation with supercritical nonlinearity

1991 Mathematics Subject Classification. 35Q53,(35B41,35B45,37L30,35L70).

Key words and phrases. Cahn-Hilliard equation, hyperbolic relaxation, generalized semiflows, global attractor, approximation of global attractors, damped semilinear wave equation.

This work was partially supported by grants of the Istituto di Matematica Applicata e Tecnologie Informatiche-CNR, Pavia, Italy. 


\section{INTRODUCTION}

In $\Omega \times(0,+\infty)$, with $\Omega$ a bounded and regular domain of $\mathbb{R}^{3}$, let us consider the following nonlinear partial differential equation

$$
\begin{gathered}
\varepsilon u_{t t}+u_{t}-\Delta(-\Delta u+\phi(u))=0, \text { for a. a. }(x, t) \in \Omega \times(0,+\infty) \\
u(x, 0)=u_{0}(x), u_{t}(x, 0)=u_{1}(x) \text { for a. a. } x \in \Omega \\
u(x, t)=\Delta u(x, t)=0, \text { for a. a. }(x, t) \in \partial \Omega \times(0,+\infty)
\end{gathered}
$$

Equation (1.1) has been introduced in [17] to describe rapid spinodal decompositions in a binary alloy occupying the region $\Omega$. We refer to the pioneering works [7], [8] and to the more recent [15] for the description of the the phenomenon and to [31] and [30] for a qualitative study of the spinodal decomposition. In this case, the unknown $u$ is a real valued function defined in $\Omega \times(0,+\infty)$ indicating the relative concentration of one component of the system. Finally, the nonlinear function $\phi$ is the derivative of a double well potential accounting for the presence of two components, e.g. $F(v)=\frac{1}{4}\left(v^{2}-1\right)^{2}$. In this physical setting, when we neglect the inertial term $u_{t t}$ (i.e. $\left.\varepsilon=0\right),(1.1)$ is the well known Cahn-Hilliard equation which plays a basic role in material science (see [7], [8], [38], [21] and references therein). The Cahn-Hilliard equation has been much studied and one has now satisfactory well posedness results as well as results on the long time behaviour of solutions (see, e.g., [13], [35], [36] and references therein). In recent years, the analysis has been dedicated also to the study of some Cahn-Hilliard type models possibly including also nonlocal terms (see the pioneering work [16] and the more recent [5] and [11]). On the contrary, less attention has been devoted to the analysis of the Cahn-Hilliard equation with the relaxation term $\varepsilon u_{t t}$. In this framework, equation (1.1) will be consequently called hyperbolic relaxation of the Cahn-Hilliard equation.

Our goal is to prove a global in time existence result and the asymptotic stability from the point of view of the global attractors for (a proper weak formulation of) (1.1). Before discussing our approach to (1.1), we prefer to make an overview of the existing results.

The first contribution to the mathematical analysis of (1.1) seems to be the one in [12]. In this work, the author proves the well posedness for the initial and boundary value problem for (1.1)-(1.3) and the existence of the maximal attractor in the one dimensional case, e.g. $\Omega=[0, L]$ moreover, the family of attractors is shown to be upper semicontinuous in $\varepsilon=0$. However, this results hold in the weak topology of the proper phase space and only for potentials $\phi$ of polinomial type. This result has been recently enhanced in [18] (but see also [6]) by proving the existences of a robust family of exponential attractors at $\varepsilon=0$ for the strongly continuous semigroup generated by (1.1). More recently, the problem of the long-time behaviour of (1.1) has been reconsidered by introducing into the equation also a viscous perturbation of the form $-\delta \Delta u_{t}$. Consequently, when both $\varepsilon$ and $\delta$ are strictly positive, equation (1.1) is named hyperbolic relaxation of the viscous CahnHilliard equation. As far as we know, the study of equation (1.1) with two 
parameters $\varepsilon$ and $\delta$ has been considered only in [33], [34], [19]. More precisely, in [33] and in [34] the authors show that (1.1), endowed with suitable boundary conditions, generates a strongly continuous semigroup on a proper phase space when both $\varepsilon$ and $\delta$ are allowed to vary in $[0,1]$. Moreover, they prove that the corresponding dynamical system is dissipative and possesses a compact attractor for which they study the regularity and the upper semi continuity at $\varepsilon=0$ and at $\delta \geq \delta_{0} \geq 0$. Finally, they construct also the exponential attractors and the inertial manifolds, for which, however, they do not discuss the stability with respect to $\varepsilon$ and $\delta$. These results hold in the one-dimensional setting and with the particular choice $\phi(v)=v^{3}-v$. Clearly, as a by product they obtain that the global attractor, being contained in the exponential attractor, is of finite fractal dimension. The three dimensional case has been recently analyzed in [19], in which, under the key assumption

$$
\varepsilon, \delta \in[0,1] \text { and } \delta \geq \lambda \varepsilon, \quad \text { for some } \lambda \in(0,1]
$$

the authors prove that (1.1) generates a strongly continuous semigroup in a certain phase space. This semigroup is shown to be dissipative and the global attractor is constructed. Moreover, (and this is certainly the main result of the paper) the problem of the existence of a robust family of exponential attractor with respect to $\varepsilon$ and $\delta$ satisfying (1.4) is addressed. As a by product, the authors obtain that the finite fractal dimension global attractor (being contained in the exponential attractor) is also stable with respect to $\varepsilon$ and $\delta$.

This paper aims to be a contribution to the problem of the long time behaviour for (1.1) in the three dimensional case without introducing any viscous term in (1.1). Thus, our result partially extends the results in [12] and [19]. From the one hand, we are able to consider the three dimensional setting and more general potential potentials $\phi$ (this extends [12]) without introducing any regularizing term as $-\Delta u_{t}$ (this extends the contribution in [19]). On the other hand however, we do not discuss any kind of stability with respect to $\varepsilon$ for the attractor we construct (this problem will be, however, addressed in a future paper) and we do not discuss the problem of the existence of exponential attracting sets. Moreover, our results hold in a phase space endowed with the weak topology (as in [12]). Thus, the resulting attractor has weaker attracting properties than the one studied in [19]. From now on we will let $\varepsilon=1$ in (1.1).

As we will show in the next sections, the main difficulty in dealing with (1.1) is the low regularity of its weak solutions (see the weak formulation 2.1 of (1.1)-(1.3)). This lack of regularity has two major consequences. First of all, it prevents from proving a uniqueness results for the solutions to (1.1). Secondly, it prevents from proving any type of energy equation for the solutions of (1.1). This fact is unpleasant since a "good" energy equation would serve in proving the asymptotic compactness of the system, even without a uniqueness result. The energy equation method for proving the asymptotic compactness was initiated by [4] and then extended and generalized in various direction (see, e.g. [37] and the references therein). These obstacles are 
bypassed in the one-dimensional setting by heavily relying of the continuous embedding $H_{0}^{1}(\Omega) \subset L^{\infty}(\Omega)$ which allows to handle the nonlinear term. On the other hand, in the three dimensional case, one needs the presence of a regularizing term as $-\delta \Delta u_{t}$ and then require that $\varepsilon$ is dominated by $\delta$, i.e., (1.4) holds. This is exactly the approach used in [19].

Here, we rely on the J.M. Ball recent theory of generalized semiflows (see [3], [4] and the next Section 3 in this paper) to handle the possible non uniqueness of solutions and we restrict our investigation to a proper subset of all the possible solutions to (1.1). More precisely, we will concentrate our attention on the set of the functions that solve (1.1) in a proper weak sense that are also limits of the Faedo-Galerkin scheme we use in proving the existence Theorem. Note that this set could be a proper subset of the set of all the solutions since, due to the non uniqueness, there might be functions solving (the weak formulation of) (1.1) which are not limits of any Faedo-Galerkin scheme. The advantage of working with this set of solutions, which will be consequently called limiting solutions, is that its elements satisfy a proper energy inequality. This fact, in particular suggest that the right topology for the phase space should be the topology induced by the weak convergence. Unfortunately, the set of solutions obtained as mentioned above fails to be a generalized semiflow. Nevertheless, by reasoning as in [40], we are able to prove that the set of the limiting solutions to (1.1) enjoys some substantial properties which allow us to prove the existence of a suitable notion of weak global attractor. Since the global attractor has been constructed for the solutions of (1.1) which can be approximated by using the aforementioned Faedo-Galerkin scheme, a natural question would be the approximability also of the global attractor of the continuous problem (1.1) with the global attractor of the Faedo-Galerkin system. In Section 6 of this paper, we provide an affirmative answer for this question by proving that the set of the solution of the Faedo-Galerkin system which approximate the solutions to (1.1) forms a generalized semiflow on a proper finite dimensional phase space. Moreover, this generalized semiflow possesses a global attractor which converges to the attractor of the continuous problem in an appropriate topology when $N$ (=the number of basis elements which are retained for the Faedo-Galerkin approximation) goes to $+\infty$. We refer the interested reader to the seminal references [22], [24], [23] and to [10] for some additional material about the problem of the upper-semicontinuity and approximability of global attractors.

It is worthwhile to note that the lacking of the energy equation for the solutions of (the weak formulation of) (1.1) is strongly reminiscent of what happens in the analysis of the nonlinear damped wave equation, when the nonlinearity $\phi$ is not locally lipschitz and has a super critical growth. In fact, let us consider in a bounded domain $\Omega \subset \mathbb{R}^{n}$ the following equation

$$
u_{t t}+u_{t}-\Delta u+\phi(u)=0 \text { in } \Omega
$$

with $\phi: \mathbb{R} \rightarrow \mathbb{R}$ a continuous function satisfying

$$
|\phi(v)| \leq C\left(1+|v|^{\gamma}\right), \quad \text { where } C>0, \quad 1 \leq \gamma<+\infty .
$$


Under some mild additional assumptions on $\phi$ one can prove the existence of at least one weak solution to (1.5) using, for instance, the Faedo-Galerkin method (see [27]). Unfortunately, it is not known whether the weak solutions to (1.5) would satisfy some energy equation (see [4]). Thus, under the unproved assumption that all the weak solutions to (1.5) satisfy the energy equation, in [4] the existence of a the global attractor in the proper phase space is proved for any $\gamma \geq 1$. However, it is easy to prove that the set of all the solutions to (1.5) constructed via the Faedo-Galerkin scheme verify a corresponding energy inequality. As for (1.1) we will prove that the set of the limiting solutions to (1.5) possesses a weak global attractor in the framework of the usual phase space for (1.5) endowed with the topology induced by the weak convergence. See [1] for another contribution in the weak topology of the phase space. See also [14] and [29], [26] for some result in the case of the super critical growth condition on $\phi$.

Plan of the paper. In the next Section 2, we recall some preliminary machinery which is needed in order to correctly set our problem. Then, we introduce the weak formulation of (1.1) we deal with. In Section 3, we recall some results on Ball's theory of generalized semiflows and present some material in the direction of studying the weak semiflow structure of the limiting solutions to (1.1) and (1.5) and the corresponding notion of weak global attractor. Section 4 contains the analysis of the Faedo Galerkin scheme approximating (1.1). Then, in Section 5 we rigorously define the concept of limiting solutions and we present the results on their long time behavior. In Section 6 we address the problem of the approximability of the global attractor for (1.1) with the one of its Faedo Galerkin system. Finally, in the last Section of the paper, we show that the results obtained for (1.1) hold also for the semilinear damped wave equation in the supercritical growth condition case.

\section{Mathematical Setting AND WEAK FORMUlation}

Function spaces Throughout the paper, we denote by $\|\cdot\|_{\mathfrak{E}}$ and $\langle\cdot, \cdot\rangle_{\mathfrak{E}}$ the norm and the scalar product in the generic Hilbert space $\mathfrak{E}$. When $\mathfrak{E}=L^{2}(\Omega)$, we omit the subscripts. We consider the positive operator

(2.1) $A=-\Delta, \quad$ with domain $D(A)=H^{2}(\Omega) \cap H_{0}^{1}(\Omega) \subset L^{2}(\Omega)$,

and the family of Hilbert spaces

$$
H^{s}=D\left(A^{s}\right), s \in \mathbb{R},
$$

with scalar product $\langle u, v\rangle_{H^{s}}:=\left\langle A^{s / 2} u, A^{s / 2} v\right\rangle$, for $u, v \in H^{s}$.

It is well known that $H^{s_{1}} \subset H^{s_{2}}$ with dense and compact immersion when $s_{1}>s_{2}$.

Then, we set

$$
\begin{gathered}
\mathscr{H}:=D\left(A^{1 / 2}\right) \times D\left(A^{-1 / 2}\right), \\
\text { with norm }\|(u, v)\|_{\mathscr{H}}^{2}:=\|u\|_{D\left(A^{1 / 2}\right)}^{2}+\|v\|_{D\left(A^{-1 / 2}\right)}^{2} .
\end{gathered}
$$

On account of the Poincarè inequality, the norm in $D\left(A^{1 / 2}\right)=H_{0}^{1}(\Omega)$ is given by $\|\nabla \cdot\|$. Finally, we let $\mathscr{H}^{*}$ to denote the topological dual of $\mathscr{H}$, 
and we denote by $\langle\langle\cdot, \cdot\rangle\rangle$ the duality pairing between $\mathscr{H}$ and $\mathscr{H}^{*}$.

In the sequel, we denote by $B_{\mathscr{H}}(r)$ the ball of radius $r$ in $\mathscr{H}$, that is

$$
B_{\mathscr{H}}(r):=\left\{(u, v) \in \mathscr{H}:\|(u, v)\|_{\mathscr{H}} \leq r\right\} .
$$

Finally, we make the following assumptions on the nonlinear function $\phi$

Conditions on $\phi$ Let $\phi \in C^{0}(\mathbb{R})$ with $\phi(0)=0$ be such that $\phi(v)=$ $F^{\prime}(v), \forall v \in \mathbb{R}$ and

$$
\begin{aligned}
& \liminf _{|u| \rightarrow+\infty} \frac{\phi(u)}{u}>-\lambda_{1}, \\
& |\phi(u)| \leq C_{0}\left(|u|^{3}+1\right),
\end{aligned}
$$

Concerning the assumptions on the nonlinear interaction term $\phi$, we note that the typical nonlinearity $\phi(v)=v^{3}-v$ is included in our framework. Note also that we do not make any type of assumption regarding the (local) Lipschitz continuity of $\phi$. Thus, a uniqueness result is not to be expected. The symbol $\lambda_{1}$ in (2.3) denotes the first eigenvalue of the Laplace operator with Dirichlet boundary conditions. Along the paper, we agree to denote by $C>0$ a generic constant which may vary from line to line and even in the same formula depending only on $\Omega$. Further dependencies of $C$ will be declared on occurrence.

We introduce the following weak formulations of the initial-boundary value problem (1.1-1.3)

Problem 2.1. For any $T>0$ and any $\left(u_{0}, u_{1}\right) \in \mathscr{H}$, find $(u, v) \in L^{\infty}(0, T ; \mathscr{H})$ with $u_{t} \in L^{2}\left(0, T ; D\left(A^{-1 / 2}\right)\right)$ such that

$$
\begin{gathered}
u_{t t}+u_{t}+A(A u+\phi(u))=0, \quad \text { in } D\left(A^{-3 / 2}\right), \text { and a.e. in }(0, T), \\
u(0)=u_{0} \quad \text { in } D\left(A^{1 / 2}\right), \quad u_{t}(0)=u_{1} \text { in } D\left(A^{-1 / 2}\right) .
\end{gathered}
$$

We show the existence of a solution to (2.5) in Theorem 5.2.

\section{Generalized Semiflows}

In this section, we briefly summarize from [3] some results concerning the theory of generalized semiflows.

Suppose we are given a metric space (not necessarily complete) $\mathcal{X}$ with metric $d_{\mathcal{X}}$. If $C$ is a subset of $\mathcal{X}$ and $b$ is point in $\mathcal{X}$, we set $\rho(b, C):=$ $\inf _{c \in C} d_{\mathcal{X}}(b, c)$, consequently, if $C \subset \mathcal{X}$ and $B \subset \mathcal{X}$, we set $\operatorname{dist}(B, C):=$ $\sup _{b \in B} \rho(b, C)$.

Definition 3.1. A generalized semiflow $\mathfrak{F}$ on $\mathcal{X}$ is a family of maps $u$ : $[0,+\infty) \rightarrow \mathcal{X}$, called solutions, satisfying the following hypotheses:

(H1) (Existence) For each $v \in \mathcal{X}$ there exists at least one $u \in \mathfrak{F}$ with $u(0)=v$.

(H2) (Translates of solutions are still solutions) If $u \in \mathfrak{F}$ and $\tau \geq 0$, then 
$u^{\tau} \in \mathfrak{F}$ where $u^{\tau}(t):=u(t+\tau), t \in(0,+\infty)$.

(H3) (Concatenation) If $u, v \in \mathfrak{F}, t \geq 0$ with $u(t)=v(0)$ then $w \in \mathfrak{F}$ where

$$
w(\tau):=\left\{\begin{array}{cl}
u(\tau) & \text { for } 0 \leq \tau \leq t \\
v(\tau-t) & \text { for } t<\tau
\end{array}\right.
$$

(H4) (Upper semi-continuity with respect to initial data) If $u_{n} \in \mathfrak{F}$ with $u_{n}(0) \rightarrow v$, then there exist a subsequence $u_{n_{k}}$ of $u_{n}$ and $u \in \mathfrak{F}$ with $u(0)=v$ such that $u_{n_{k}}(t) \rightarrow u(t)$ for each $t \geq 0$.

Furthermore, a generalized semiflow can satisfy (or not) the following continuity properties.

(C1) Each $u \in \mathfrak{F}$ is continuous from $(0,+\infty)$ to $\mathcal{X}$.

(C2) If $u_{n} \in \mathfrak{F}$ with $u_{n}(0) \rightarrow v$, then there exists a subsequence $u_{n_{k}}$ of $u_{n}$ and $u \in \mathfrak{F}$ with $u(0)=v$ such that $u_{n_{k}}(t) \rightarrow u(t)$ uniformly for $t$ in compact subsets of $(0,+\infty)$.

(C3) Each $u \in \mathfrak{F}$ is continuous from $[0,+\infty)$ to $\mathcal{X}$.

(C4) If $u_{n} \in \mathfrak{F}$ with $u_{n}(0) \rightarrow v$, then there exists a subsequence $u_{n_{k}}$ of $u_{n}$ and $u \in \mathfrak{F}$ with $u(0)=v$ such that $u_{n_{k}}(t) \rightarrow u(t)$ uniformly for $t$ in compact subsets of $[0,+\infty)$.

When the phase space $\mathcal{X}$ is a Banach space, we can introduce the analogous of $(C 4)$ in the weak topology, namely :

$(C 4)_{w}$ If $u_{n} \in \mathfrak{F}$ with $u_{n}(0) \rightarrow v$ in $\mathcal{X}$, then there exists a subsequence $u_{n_{k}}$ of $u_{n}$ and $u \in \mathfrak{F}$ with $u(0)=v$ such that $u_{n_{k}}(t) \rightarrow u(t)$ in $\mathcal{X}$ and uniformly for $t$ in compact subsets of $[0,+\infty)$.

For other interesting properties on generalized semiflows, especially relating measurability and continuity we refer to [3]. These results are extension to generalized semiflows of the results of [2] concerning semiflows originally given for nonlinear evolutionary processes on metric spaces .

It is possible to extend to generalized semiflow the standard definition concerning absorbing sets and attractors given for semiflows and semigroups (cf. [41] and [42]) . Let $\mathfrak{F}$ be a generalized semiflow and let $E \subset \mathcal{X}$. For any $t \geq 0$, we define

$$
T(t) E=\{u(t): u \in \mathfrak{F} \text { with } u(0) \in E\},
$$

so that $T(t): 2^{\mathcal{X}} \rightarrow 2^{\mathcal{X}}$, denoting by $2^{\mathcal{X}}$ the space of all subsets of $\mathcal{X}$. It is worthwhile to note that, thanks to $(H 2)$ and $(H 3),\{T(t)\}_{t \geq 0}$ defines a semigroup on $2^{\mathcal{X}}$. On the other hand, $(H 4)$ implies that $T(t) z$ is compact for any $z \in \mathcal{X}$.

The the positive orbit of $u \in \mathfrak{F}$ is the set $\gamma^{+}(u)=\{u(t): t \geq 0\}$. If $E \subset \mathcal{X}$ then the positive orbit of $E$ is the set

$$
\gamma^{+}(E)=\bigcup_{t \geq 0} T(t) E=\bigcup\left\{\gamma^{+}(u): u \in \mathfrak{F}, u(0) \in E\right\} .
$$

The $\omega$-limit set of $u \in \mathfrak{F}$ is the set

$$
\omega(u)=\left\{v \in X: u\left(t_{n}\right) \rightarrow v \text { for some sequence } t_{n} \nearrow+\infty\right\},
$$


while the $\omega$ - limit set of $E$, is the set

$$
\begin{gathered}
\omega(E):=\left\{u^{\infty} \in \mathcal{X}: \text { there exist } u_{n} \in \mathfrak{F} \text { with } u_{n}(0) \in E, u_{n}(0)\right. \text { bounded } \\
\text { and a sequence } \left.t_{n} \nearrow+\infty \text { with } u_{n}\left(t_{n}\right) \rightarrow u^{\infty}\right\}
\end{gathered}
$$

A complete orbit is a map $\psi: \mathbb{R} \rightarrow \mathcal{X}$ such that, for any $s \in \mathbb{R}, \psi^{s} \in \mathfrak{F}$. Then, if $\psi$ is a complete orbit, we can define the $\alpha$-limit set of $\psi$ as

$$
\alpha(\psi):=\left\{z \in \mathcal{X}: \psi\left(t_{n}\right) \rightarrow z \text { for some sequence } t_{n} \rightarrow-\infty\right\} .
$$

We say that the subset $\mathcal{U} \subset \mathcal{X}$ attracts a set $E$ if $\operatorname{dist}(T(t) E, \mathcal{U}) \rightarrow 0$ as $t \rightarrow+\infty$.

We say that $\mathcal{U}$ is positively invariant if $T(t) \mathcal{U} \subset \mathcal{U}$ for all $t \geq 0$, while $\mathcal{U}$ is invariant if $T(t) \mathcal{U}=\mathcal{U}$ for all $t \geq 0$. Finally, we say that $\mathcal{U}$ is quasi invariant if for any $z \in \mathcal{U}$ there exists a complete orbit $\psi$ with $\psi(0)=z$ and $\psi(t) \in \mathcal{U}$ for any $t \in \mathbb{R}$. Note that if $\mathcal{U}$ is quasi invariant then there holds $\mathcal{U} \subset T(t) \mathcal{U}$ for all $t \geq 0$. Finally, a subset $\mathcal{U}$ will be declared invariant if and only if is positively invariant and quasi invariant.

The subset $\mathscr{A} \subset \mathcal{X}$ is a global attractor if $\mathscr{A}$ is compact, invariant, and attracts all bounded sets.

$\mathfrak{F}$ is eventually bounded if, given any bounded $B \subset \mathcal{X}$, there exists $\tau \geq 0$ with $\gamma^{\tau}(B)$ bounded.

$\mathfrak{F}$ is point dissipative if there exists a bounded set $B_{0}$ such that, for any $u \in \mathfrak{F}, u(t) \in B_{0}$ for all sufficiently large $t \geq 0$.

$\mathfrak{F}$ is asymptotically compact if for any sequence $u_{n} \in \mathfrak{F}$ with $u_{n}(0)$ bounded, and for any sequence $t_{n} \nearrow+\infty$, the sequence $u_{n}\left(t_{n}\right)$ has a convergent subsequence.

We will also make use of the notion of Lyapunov function, which can be introduced starting from the following definitions: we say that a complete orbit $g \in \mathfrak{F}$ is stationary if there exists $x \in \mathcal{X}$ such that $g(t)=x$ for all $t \in \mathbb{R}-\operatorname{such} x$ is then called a rest point. Note that the set of rest points of the semiflow $\mathfrak{F}$, denoted by $\mathcal{Z}(\mathfrak{F})$, is closed in view of $(\mathrm{H} 4)$. A function $V: \mathcal{X} \rightarrow \mathbb{R}$ is said to be a Lyapunov function for $\mathfrak{F}$ provided:

(i) $\quad V$ is continuous

(ii) $V(g(t)) \leq V(g(s))$ for all $g \in \mathfrak{F}$ and $0 \leq s \leq t$ (i.e., $V$ decreases along solutions)

(iii) if the map $t \mapsto V(g(t))$ is constant for some complete orbit $g$, then $g$ is a stationary orbit

Finally, we say that a global attractor $\mathscr{A}$ for $\mathfrak{F}$ is Lyapunov stable if for any $\varepsilon>0$ there exists $\delta>0$ such that for any $E \subset \mathfrak{F}$ with $e(E, \mathscr{A}) \leq \delta$, then $e(T(t) E, \mathscr{A}) \leq \varepsilon$ for all $t \geq 0$.

We now quote the two general abstract criterion providing a sufficient and necessary condition for the existence of the attractor (see [3, Thms 3.3, 5.1 , and 6.1].

Theorem 3.2. A generalize semiflow $\mathfrak{F}$ has a global attractor if and only if $\mathfrak{F}$ is point dissipative and asymptotically compact. The attractor $\mathscr{A}$ is unique. Moreover, $\mathscr{A}$ is the maximal compact invariant set of $\mathcal{X}$ and it is 
given by

$$
\mathscr{A}=\bigcup\{\omega(B): \quad B \quad \text { a bounded set of } \mathcal{X}\}=\omega(\mathcal{X})
$$

Besides, if $\mathfrak{F}$ complies with $(C 1)$ and $(C 4)$, then $\mathscr{A}$ it is also Lyapunov stable.

Moreover, when the generalized semiflow admits a Lyapunov function, there holds the following

Theorem 3.3. Assume that $\mathfrak{F}$ is asymptotically compact, admits a Lyapunov function $V$, and that the sets of its rest points $\mathcal{Z}(\mathfrak{F})$ is bounded. Then, $\mathfrak{F}$ is also point dissipative, and thus admits a global attractor $\mathscr{A}$. Moreover, $\omega(u) \subset \mathcal{Z}(\mathfrak{F})$ for all trajectories $u \in \mathfrak{F}$.

3.1. A weaker notion of attractor. The possible application of the above reported abstract theory to suitable classes of differential problems is often delicate. Indeed, one is usually forced to carefully choose the correct notion of solution of the problem, in order to possibly check the validity of properties (H1)-(H4). This process may not be straightforward whenever one considers some suitably weak notion of solvability. On the one hand, solutions have indeed to be weak enough in order to fulfill (H1) (the assumption (H2) is generally easy to meet in actual situations). On the other hand, the notion of solution has to be robust enough in order to fulfill (H4). This robustness may turn out to be in conflict with (H3).

For instance, the existence of weak solutions to a differential problem is usually proved by approximation (like, e.g., for the solutions of equation (2.5) cf. Definition 5.1 and Theorem 5.2). Then, one is naturally led to define the candidate semiflow as the set of all solutions which are limits in a suitable topology of their approximate solutions. Axioms (H1) and (H2) will be trivially checked, and, if the aforementioned topology is strong enough, one can hopefully verify (H4) as well. However, due to this approximation procedure, the concatenation in (H3) may not hold (the approximating sequences may not have the same indices), as in the case of the set of limiting weak solutions to (2.5).

To overcome this difficulty, in [40] was observed that it is possible to partially extend Theorem 3.2 to the case of a non-empty set $\mathcal{G}$ of functions $g:[0,+\infty) \rightarrow \mathcal{X}$, complying with (H1), (H2), (H4), but not necessarily (H3) (see the Definition 3.4 below). In particular, the mapping $T$ constructed from $\mathcal{G}$ as in (3.1) fails to be a semigroup and fulfills instead the following

$$
T(t+s) B \subset T(t) T(s) B \quad \forall s, t \geq 0, B \subset X .
$$

The price of dropping the semigroup property for $T$ consists in the fact that the weak attractor notion introduced below will be quasi-invariant but will fail to be invariant.

We now introduce the concept of weak generalized semiflow

Definition 3.4 (Weak generalized semiflow). A non-empty set of maps $g$ : $[0,+\infty) \rightarrow \mathcal{X}$ fulfilling $(H 1),(H 2)$ and $(H 4)$ will be named weak generalized semiflow.

Correspondingly, we introduce a weaker notion of attractor as follows 
Definition 3.5 (Weak global attractor). Let $\mathcal{G}$ be a weak generalized semiflow. We say that a non-empty set $\mathscr{A}$ is a weak global attractor for $\mathcal{G}$ if it is compact, quasi-invariant, and attracts all the bounded sets of $\mathcal{X}$.

Moreover, we shall observe that a weak global attractor (if existing), is minimal in the set of closed subsets of $X$ attracting all bounded sets, hence unique, cf. [32]. We may state the following

Theorem 3.6. Let $\mathcal{G}$ be a non-empty set of functions $g:[0,+\infty) \rightarrow \mathcal{X}$ complying with (H1), (H2) and (H4). Moreover, assume that $\mathcal{G}$ is asymptotically compact and point dissipative. Then, $\mathcal{G}$ possesses a weak global attractor $\mathcal{A}$. Moreover, the attractor $\mathscr{A}$ enjoys the following description

(3.4) $\mathscr{A}=\{\xi \in \mathcal{X}:$ there exists a bounded complete orbit $z: z(0)=\xi\}$.

The proof of the existence of the weak global attractor follows the proof of Theorem 3.2. For the sake of completeness, we outline below the proof of this result, which actually consists in checking that the argument developed in [3] for the proof of Theorem 3.2 goes through without the concatenation condition (H3) (see also [40]). The property (3.4) is well known for attractors of semigroups (cf. [42]), for uniform attractors for semiprocesses (cf. [10]) and also for generalized multivalued semigroups (see [39]). Since we did not find in the literature the proof for this property in the generalized semiflow framework, we report it. Our argument closely follows the proof of the corresponding property for semigroups. Clearly, the same property holds for the attractor constructed in Theorems 3.2 and 3.3.

Proof. Following the proof of [3, Thm. 3.3], one has to preliminarily show these two facts: their proof simply consists in repeating the arguments of [3, Lemmas 3.4, 3.5], which are valid independently of (H3).

Claim 1.: If $\mathcal{G}$ fulfills $(\mathrm{H} 1),(\mathrm{H} 2),(\mathrm{H} 4)$, and is asymptotically compact, then for any non-empty and bounded set $B \subset X, \omega(B)$ is non-empty, compact, quasi-invariant and attracts $B$.

Claim 2.: If $\mathcal{G}$ fulfills $(\mathrm{H} 1),(\mathrm{H} 2),(\mathrm{H} 4)$, is asymptotically compact and point dissipative, then there exists a bounded set $\mathcal{B}$ such that for any compact $K \subset \mathcal{X}$ there exist $\tau=\tau(K)>0$ and $\varepsilon=\varepsilon(K)>0$ with $T(t)\left(N_{\varepsilon}(K)\right) \subset \mathcal{B}$ for all $t \geq t_{1}$.

Hence, let us define $\mathscr{A}:=\omega(\mathcal{B})$ where $\mathcal{B}$ is exactly the bounded set of Claim 2. Owing to Claim $1, \mathscr{A}$ is non-empty, compact, quasi-invariant, and attracts $\mathcal{B}$. Let us now fix any bounded set $B$ and consider its compact $\omega$ limit $K:=\omega(B)$, which attracts $B$ by Claim 1. Using Claim 2, one readily exploits (3.3) and adapts the proof of [3, Thm. 3.3] in order to infer that $\mathcal{B}$ attracts $B$ as well. Thus, also $\mathcal{A}$ attracts $B$ and, being $B$ arbitrary among bounded sets, we have checked that $\mathcal{A}$ is the weak global attractor.

Now we prove (3.4). To this end, we take $\xi \in \mathscr{A}$. Then, the quasi invariance of $\mathscr{A}$ entails that there exists a complete orbit $z$ such that $z(0)=\xi$ and $z(t) \in$ $\mathscr{A}$ for any $t$. In particular, $z$ is also bounded since $\mathscr{A}$ is bounded. Let us now prove the reverse inclusion. Consider any bounded and complete orbit $z$ in $\mathcal{G}$ and then set $\mathcal{O}:=\{z(t), \quad t \in \mathbb{R}\}$. Now, the set $\mathcal{O}$ is clearly bounded in the phase space and quasi invariant, thus there holds the following chain 
of inclusions

$$
\mathcal{O} \subset T(t) \mathcal{O} \subset \omega(\mathcal{O}) \subset \mathscr{A} .
$$

In particular, the first inclusion holds by virtue of the quasi invariance of $\mathcal{O}$, while the second one holds since the $\omega$-limit set of any bounded set attracts the set itself, Finally, the last inclusion easily follows from (3.2). Thus, we conclude that for any bounded and complete orbit $z$ of $\mathcal{G}$, there holds $z(0) \in \mathscr{A}$. This fact clearly implies (3.4).

\section{Faedo Galerkin approximation of the hyperbolic Relaxation OF THE CAHN-Hilliard EQUATION}

In this section, we perform a detailed analysis of the Faedo-Galerkin scheme approximating (2.5). This analysis has two major scope. First, the Faedo-Galerkin scheme we are going to introduce is the starting point in proving the existence of solutions to (2.5). Moreover, the solutions to (2.5) that can be obtained as limits of this approximation argument (see 5.1) are the ones for which we will prove the existence of a (suitable weak notion of ) attractor. Secondly, we prove that the set of all the solutions to the Faedo-Galerkin system forms a generalized semiflow in a proper finite dimensional phase space. For this generalized semiflow we show in this section the existence of the global attractor $\mathscr{A}_{N}$.

Since $A^{-1}$ is compact and self-adjoint there exists an orthonormal basis of $L^{2}(\Omega)$ consisting of eigenvectors of $A$, that is

$$
\begin{gathered}
A v_{i}=\lambda_{i} v_{i}, \quad\left\|v_{i}\right\|=1, \text { for } i=1,2, \ldots \\
\quad\left\langle v_{i}, v_{j}\right\rangle=0, \text { for } i \neq j \\
0<\lambda_{1} \leq \lambda_{2} \leq \ldots, \lambda_{j} \rightarrow+\infty \text { as } j \nearrow+\infty .
\end{gathered}
$$

It is well known that $\left\{\lambda_{n}^{-1 / 2} v_{n}\right\}_{n \geq 1}$ is an orthonormal basis of $D\left(A^{1 / 2}\right)$. For any $N \geq 1$ we let $V^{N}$ to be the space spanned by $\left\{v_{1}, \ldots, v_{N}\right\}$. We consider the following equation in $V^{N}$.

Problem $_{N}$ : Find $t_{N}$ and $u_{i} \in C^{2}\left(\left[0, t_{N}\right]\right), i=1, \ldots, N$ such that, if

$$
u^{n}:=\sum_{i=1}^{N} u_{i}(t) v_{i},
$$

then $u^{n} \in C^{2}\left(\left[0, t_{N}\right], V_{N}\right)$ solves $(i=1, \ldots, N)$

$$
\begin{aligned}
\left\langle u_{t t}^{N}, v_{i}\right\rangle+\left\langle u_{t}^{N}, v_{i}\right\rangle & +\left\langle A\left(A u^{N}+\phi\left(u^{N}\right)\right), v_{i}\right\rangle=0, \\
\left\langle u^{N}(0)-u_{0}^{N}, v_{i}\right\rangle & =0, \quad\left\langle u_{t}^{N}(0)-u_{1}^{N}, v_{i}\right\rangle=0,
\end{aligned}
$$

where $\left(u_{0}^{N}, u_{1}^{N}\right)$ belongs to the discrete space $V^{N} \times V^{N}$. This problem consist in a $N$-dimensional system of nonlinear ordinary differential equations, for which standard methods show that there exists $t_{N} \in(0,+\infty)$ such that (4) has at least a solution in $\left[0, t_{N}\right]$. Note that, also at this approximating level the uniqueness of the solution is not to be expected, since $\phi$ is only continuous and not locally Lipschitz. The subsequent fundamental a priori estimate will show that the approximating solution are actually defined over 
all $(0,+\infty)$. This a priori estimate will be crucial in order to pass to the limit as $N \rightarrow+\infty$ and to obtain a solution to (2.5) (see the forthcoming section 5)

Main Estimate Multiply (4.2) by $u_{i}^{\prime}(t) / \lambda_{i}$ and then sum over $i$. We get

$$
\frac{d}{d t} V\left(u^{N}, u_{t}^{N}\right)(t)=-\left\|u_{t}^{N}(t)\right\|_{D\left(A^{-1 / 2}\right)}^{2}, \quad \forall t,
$$

which imply

$$
\begin{aligned}
& V\left(u^{N}(t), u_{t}^{N}(t)\right)+\int_{s}^{t}\left\|u_{t}^{N}(\lambda)\right\|_{D\left(A^{-1 / 2}\right)}^{2} d \lambda \\
& =V\left(u^{N}(s), u_{t}^{N}(s)\right), \quad \forall t, s \in[0,+\infty),
\end{aligned}
$$

where we have set

$$
V\left(u^{N}, u_{t}^{N}\right)(t):=\frac{1}{2}\left\|u_{t}^{N}(t)\right\|_{D\left(A^{-1 / 2}\right)}^{2}+\frac{1}{2}\left\|\nabla u^{N}(t)\right\|^{2}+\int_{\Omega} F\left(u^{N}(t)\right) .
$$

Now, since (2.3) entails that

$$
F(u) \geq-\frac{\lambda}{2} u^{2}+C,
$$

for some $\lambda<\lambda_{1}$ and some $C$, we have that

$$
V\left(u^{N}, u_{t}^{N}\right) \geq C\left\|\left(u^{N}, u_{t}^{N}\right)\right\|_{\mathscr{H}}-C .
$$

Estimate (4.5) has some interesting and important consequences. First of all it shows that the discrete solution can be extended over all the positive line $(0,+\infty)$ and moreover that the solutions emanating from bounded sets of initial data remain bounded, even independently of $N$. In fact, by combining (4.5) with $s=0$ and (4.8), we have that

$$
\left\|\left(u^{N}(t), u_{t}^{N}(t)\right)\right\|_{\mathscr{H}} \leq C(R), \forall t>0,
$$

as soon as $\left\|\left(u^{N}(0), u_{t}^{N}(0)\right)\right\|_{\mathscr{H}} \leq R$.

Estimate (4.5) is also the starting point to show that the set of all the solution at level $N$ of the approximating equation (4.2) is a generalized semiflow $\mathcal{E}_{N}$ in the phase space $V^{N} \times V^{N}$ endowed with the topology induced by $D\left(A^{1 / 2}\right) \times D\left(A^{-1 / 2}\right)$, which is point dissipative, and asymptotically compact, thus admitting a global attractor.

The first step consist in showing that the set $\mathcal{E}_{N}$ of all the solutions to (4.2) satisfies the hypothesis $(H 1)-(H 4)$ in the definition of generalized semiflow. Clearly, the existence $(H 1)$, the concatenation $(H 2)$ and the translation $(H 3)$ are immediate. To verify $(H 4)$ we have to fix a sequence $\left(u_{k 0}^{N}, u_{k 1}^{N}\right)$ in $V^{N} \times V^{N}$ converging as $k \nearrow+\infty$, with respect to the topology induced by $D\left(A^{1 / 2}\right) \times D\left(A^{-1 / 2}\right)$, to $\left(u_{0}^{N}, u_{1}^{N}\right) \in V^{N} \times V^{N}$. Then we have to show that the corresponding solutions $u_{k}^{N}$ emanating from $\left(u_{k 0}^{N}, u_{k 1}^{N}\right)$ converge (up to the extraction of a subsequence) to a function $u^{N}$ which solves (4.2) with 
$\left(u^{N}(0), u_{t}^{N}(0)\right)=\left(u_{0}^{N}, u_{1}^{N}\right)$. The convergence holding in the following sense

$$
\begin{gathered}
u_{k}^{N}(t) \rightarrow u^{N}(t) \quad \text { in } D\left(A^{1 / 2}\right) \text { and } \forall t \in[0,+\infty) \\
u_{k t}^{N}(t) \rightarrow u_{t}^{N}(t) \quad \text { in } D\left(A^{-1 / 2}\right) \text { and } \forall t \in[0,+\infty)
\end{gathered}
$$

We start by proving that (4.10)-(4.11) hold in the weak topologies of $D\left(A^{1 / 2}\right)$ and $D\left(A^{-1 / 2}\right)$, respectively. To this end, we first note that the energy equation (4.5), (4.8) and standard weak star compactness results combined with the growth conditions on $\phi$ (and Lemma 3.3 in [4]) entail that there exist $u^{N} \in W^{1, \infty}\left(0, T ; V^{N}\right), \forall T>0$ and a subsequence of $k$ (not relabeled) such that

$$
\begin{gathered}
u_{k}^{N} \stackrel{*}{\rightarrow} u^{N} \text { in } L^{\infty}\left(0, T ; D\left(A^{1 / 2}\right)\right), \quad \forall T>0 \text { as } k \nearrow+\infty, \\
u_{k t}^{N} \stackrel{*}{\rightarrow} u_{t}^{N} \text { in } L^{\infty}\left(0, T ; D\left(A^{-1 / 2}\right)\right), \quad \forall T>0 \text { as } k \nearrow+\infty, \\
\phi\left(u_{k}^{N}\right) \stackrel{*}{\rightarrow} \phi\left(u^{N}\right) \text { in } L^{\infty}\left(0, T ; L^{2}(\Omega)\right), \quad \forall T>0 \text { as } k \nearrow+\infty .
\end{gathered}
$$

Moreover, by multiplying (4.2) with $u_{k i}^{\prime \prime}(t) \lambda_{i}{ }^{-3}$ and then summing over $i$ (which correspond to testing with $A^{-3} u_{k t t}^{N}$ ), we obtain

$$
\begin{aligned}
\left\|A^{-3 / 2} u_{k t t}^{N}(t)\right\|^{2} & \leq\left\langle A^{-3 / 2} u_{k t}^{N}(t), A^{-3 / 2} u_{k t t}^{N}(t)\right\rangle+\left\langle A^{1 / 2} u_{k}^{N}(t), A^{-3 / 2} u_{k t t}^{N}(t)\right\rangle \\
(4.15) & +\left\langle A^{-1 / 2} \phi\left(u_{k}^{N}(t)\right), A^{-3 / 2} u_{k t t}^{N}(t)\right\rangle \forall t>0,
\end{aligned}
$$

and thus, recalling (4.5) and the continuous embeddings $L^{2}(\Omega) \hookrightarrow D\left(A^{-1 / 2}\right) \hookrightarrow$ $D\left(A^{-3 / 2}\right)$, we get

$$
\left\|u_{k t t}^{N}\right\|_{L^{\infty}\left(0, T ; D\left(A^{-3 / 2}\right)\right)}^{2} \leq C,
$$

which implies (again, up to the extraction of a subsequence of $k$ ) that

$$
u_{k t t}^{N} \stackrel{*}{\rightarrow} u_{t t}^{N} \text { in } L^{\infty}\left(0, T ; D\left(A^{-3 / 2}\right)\right), \forall T>0 \text { as } k \nearrow+\infty .
$$

Convergences (4.12)-(4.14) and (4.17) show that $u^{N}$ is a solution to (4.2) with $\left(u^{N}(0), u_{t}^{N}(0)\right)=\left(u_{0}^{N}, u_{1}^{N}\right)$. Note in particular, that the limit function $u^{N}$, being a solution of (4.2) verifies the energy equality (4.5). Thus we have that $\left(u^{N}, u_{t}^{N}\right) \in C^{0}([0, T] ; \mathscr{H})$. Now, we fix a $t$ in $[0, T]$ and we choose a sequence $t_{k} \rightarrow t$. Since there holds

$$
u_{k}^{N}\left(t_{k}\right)=u_{k}^{N}(0)+\int_{0}^{t_{k}} u_{k t}^{N}(s) d s, \text { in } D\left(A^{-1 / 2}\right),
$$

we have,

$$
\begin{gathered}
\left\langle A^{-1 / 2} u_{k}^{N}\left(t_{k}\right), A^{1 / 2} v\right\rangle \\
=\left\langle A^{-1 / 2} u_{k}^{N}(0), A^{1 / 2} v\right\rangle+\int_{0}^{t_{k}}\left\langle A^{-1 / 2} u_{k t}^{N}(s), A^{1 / 2} v\right\rangle d s \\
\rightarrow\left\langle A^{-1 / 2} u^{N}(0), A^{1 / 2} v\right\rangle+\int_{0}^{t}\left\langle A^{-1 / 2} u_{t}^{N}(s), A^{1 / 2} v\right\rangle d s \\
=\left\langle A^{-1 / 2} u^{N}(t), A^{1 / 2} v\right\rangle, \forall v \in D\left(A^{1 / 2}\right),
\end{gathered}
$$

thanks to the convergence $u_{k t}^{N} \stackrel{*}{\rightarrow} u_{t}^{N}$ in $L^{\infty}\left(0, T ; D\left(A^{-1 / 2}\right)\right)$. This convergence means $u_{k}^{N}\left(t_{k}\right) \rightarrow u^{N}(t)$ in $L^{2}(\Omega)$ which implies $u_{k}^{N}\left(t_{k}\right) \rightarrow u^{N}(t)$ in $H_{0}^{1}(\Omega)$ by density and (4.12). The same reasoning (using this time the convergence (4.17)), shows the analogous convergence for $u_{k t}^{N}$, namely 
$u_{k t}^{N}\left(t_{k}\right) \rightarrow u_{t}^{N}(t)$ in $D\left(A^{-1 / 2}\right)$. In particular, these facts entail that $\mathcal{E}_{N}$ satisfies $(C 4)_{w}$.

Now, since $V$ in (4.6) is lower semicontinuous, we have that

$$
\begin{aligned}
V\left(\left(u^{N}(t), u_{t}^{N}(t)\right)\right. & \leq \liminf _{k \nearrow+\infty} V\left(u_{k}^{N}\left(t_{k}\right), u_{k t}^{N}\left(t_{k}\right)\right), \\
\int_{0}^{t}\left\|u_{t}^{N}(\lambda)\right\|_{D\left(A^{-1 / 2}\right)}^{2} d \lambda & \leq \liminf _{k \nearrow+\infty}^{t_{k}}\left\|u_{0}^{N}(\lambda)\right\|_{D\left(A^{-1 / 2}\right)}^{2} d \lambda .
\end{aligned}
$$

Hence we have

$$
\begin{gathered}
V\left(\left(u^{N}(t), u_{t}^{N}(t)\right)+\int_{0}^{t}\left\|u_{t}^{N}(\lambda)\right\|_{D\left(A^{-1 / 2}\right)}^{2} d \lambda\right. \\
\leq \liminf _{k \nearrow+\infty}\left(V\left(u_{k}^{N}\left(t_{k}\right), u_{k t}^{N}\left(t_{k}\right)\right)+\int_{0}^{t_{k}}\left\|u_{k t}^{N}(\lambda)\right\|_{D\left(A^{-1 / 2}\right)}^{2} d \lambda\right),
\end{gathered}
$$

entailing in particular that $V\left(u_{k}^{N}\left(t_{k}\right), u_{k t}^{N}\left(t_{k}\right)\right) \rightarrow V\left(\left(u^{N}(t), u_{t}^{N}(t)\right.\right.$ in $\mathbb{R}$, since the both sides of (4.21) are equal to $V\left(u^{N}(0), u_{t}^{N}(0)\right)$. We can thus conclude that $u_{k}^{N}\left(t_{k}\right) \rightarrow u^{N}(t)$ in $H_{0}^{1}(\Omega)$ and $u_{k t}^{N}\left(t_{k}\right) \rightarrow u_{t}^{N}(t)$ in $D\left(A^{-1 / 2}\right)$, which clearly implies $u_{k}^{N} \rightarrow u^{N}$ in $H_{0}^{1}(\Omega)$ and $u_{k t}^{N} \rightarrow u_{t}^{N}$ in $D\left(A^{-1 / 2}\right)$, uniformly for $t \in[0, T], \forall T>0$. Thus, we obtain the desired upper semicontinuity $(H 4)$, which concludes the proof of the fact that the set $\mathcal{E}_{N}$ of the solutions to (4.2) is a generalized semiflow on $V^{N} \times V^{N}$.

We now prove that the generalized semiflow $\mathcal{E}^{N}$ is asymptotically compact and point dissipative, hence, following Ball, it possesses a global attractor $\mathscr{A}_{N}$.

Asymptotic compactness To prove the asymptotic compactness of $\mathcal{E}_{N}$ we use the so-called energy method, inaugurated by J.M.Ball and in particular we follow directly the analysis of Ball in [4]. The only (minor) difference lies in the choice of the energy functional. Here, we choose

$$
I\left(u^{N}, u_{t}^{N}\right)(t):=V\left(u^{N}, u_{t}^{N}\right)(t)+\frac{1}{2}\left\langle A^{-1 / 2} u^{N}(t), A^{-1 / 2} u_{t}^{N}(t)\right\rangle .
$$

By simply multiplying (4.2) by $\frac{1}{\lambda_{i}}\left(u_{i}^{\prime}(t)+\frac{1}{2} u_{i}(t)\right)$ and then sum over $i$, one can show that $I$ satisfies the following equation

$$
\frac{d}{d t} I\left(u^{N}, u_{t}^{N}\right)(t)=-I\left(u^{N}, u_{t}^{N}\right)(t)+\int_{\Omega}\left(F\left(u^{N}(t)\right)-\frac{1}{2} u^{N}(t) \phi\left(u^{N}(t)\right)\right) .
$$

With (4.22) and (4.23) in hand, the proof of the asymptotic compactness of $\mathcal{E}_{N}$ goes exactly like the one given by Ball in [4]. For the reader's convenience we give a sketch of the proof.

We let $\left(u_{j}^{N}, u_{j t}^{N}\right) \in \mathcal{E}_{N}$ with $\left(u_{j}^{N}(0), u_{j t}^{N}(0)\right)$ bounded and we let $t_{j} \nearrow+\infty$. Now, the energy equation (4.5) shows that $V\left(u_{j}^{N}\left(t_{j}\right), u_{j t}^{N}\left(t_{j}\right)\right)$ is bounded and thus, by (4.8), so is $\left(u_{j}^{N}\left(t_{j}\right), u_{j t}^{N}\left(t_{j}\right)\right)$ in $D\left(A^{1 / 2}\right) \times D\left(A^{-1 / 2}\right)$. Thus, up to the extraction of a subsequence of $j$, we have that $\left(u_{j}^{N}\left(t_{j}\right), u_{j t}^{N}\left(t_{j}\right)\right) \rightarrow \xi$ and also $\left(u_{j}^{N}\left(t_{j}-M\right), u_{j t}^{N}\left(t_{j}-M\right)\right) \rightarrow \xi_{-M}$, with $\xi$ and $\xi_{-M} \in \mathscr{H}$. Now, since $\mathcal{E}_{N}$ enjoys $(C 4)_{w}$, we can assume that there exists $\left(u^{N}, u_{t}^{N}\right) \in \mathcal{E}_{N}$ such 
that

$$
\left(u_{j}^{N}\left(t_{j}-M+t\right), u_{j t}^{N}\left(t_{j}-M+t\right)\right) \rightarrow\left(u^{N}, u_{t}^{N}\right)
$$

and $\left(u^{N}(0), u_{t}^{N}(0)\right)=\xi_{-M},\left(u^{N}(M), u_{t}^{N}(M)\right)=\xi$. Now, we write (4.23) integrated between 0 and $M$ for $\left(u_{j}^{N}\left(t_{j}-M+t\right), u_{j t}^{N}\left(t_{j}-M+t\right)\right)$ obtaining

$$
\begin{gathered}
I\left(u_{j}^{N}, u_{j t}^{N}\right) \\
=e^{-M} I\left(u_{j}^{N}\left(t_{j}-M\right), u_{j t}^{N}\left(t_{j}-M\right)\right)+\int_{0}^{M} e^{t-M} \int_{\Omega}\left(F\left(u_{j}^{N}\right)-\frac{1}{2} u_{j}^{N} \phi\left(u_{j}^{N}\right)\right) d t .
\end{gathered}
$$

Now, the energy estimate (4.5) and the growth hypothesis on $\phi$ (hence on $F)$ entail that $\int_{\Omega}\left(F\left(u_{j}^{N}\right)-\frac{1}{2} u_{j}^{N} \phi\left(u_{j}^{N}\right)\right)$ is uniformly bounded on the interval $[0, M]$ and moreover

$$
\int_{\Omega}\left(F\left(u_{j}^{N}(t)\right)-\frac{1}{2} u_{j}^{N}(t) \phi\left(u_{j}^{N}(t)\right) \rightarrow \int_{\Omega}\left(F\left(u^{N}(t)\right)-\frac{1}{2} u^{N}(t) \phi\left(u^{N}(t)\right) .\right.\right.
$$

Thus, we have

$$
\begin{gathered}
\lim _{j \nearrow+\infty} \int_{0}^{M} e^{t-M} \int_{\Omega}\left(F\left(u_{j}^{N}(t)\right)-\frac{1}{2} u_{j}^{N}(t) \phi\left(u_{j}^{N}(t)\right) d t\right. \\
=\int_{0}^{M} e^{t-M} \int_{\Omega}\left(F\left(u^{N}(t)\right)-\frac{1}{2} u^{N}(t) \phi\left(u^{N}(t)\right) d t .\right.
\end{gathered}
$$

Now, by writing (4.23) integrated between 0 and $M$ for $\left(u^{N}, u_{t}^{N}\right)$, we have that

$$
\lim _{j \nearrow+\infty} \int_{0}^{M} e^{t-M} \int_{\Omega}\left(F\left(u_{j}^{N}(t)\right)-\frac{1}{2} u_{j}^{N}(t) \phi\left(u_{j}^{N}(t)\right) d t=I(\xi)-e^{-M} I\left(\xi_{-M}\right) .\right.
$$

Finally, by the lower semicontinuity of $I$ and by letting $M \nearrow+\infty$ we discover that

$$
\limsup _{j \nearrow+\infty} I\left(u_{j}^{N}, u_{j t}^{N}\right) \leq I(\xi) \leq \liminf _{j \nearrow+\infty} I\left(u_{j}^{N}, u_{j t}^{N}\right),
$$

which implies that $\left\|\left(u_{j}^{N}, u_{j t}^{N}\right)\right\|_{\mathscr{H}} \rightarrow\|\xi\|_{\mathscr{H}}$ and thus $\left(u_{j}^{N}, u_{j t}^{N}\right) \rightarrow \xi$ strongly in $\mathscr{H}$. This proves the asymptotic compactness of $\mathcal{E}_{N}$.

Point dissipativity We start by proving that the functional $V$ in (4.6) is indeed the Lyapunov functional for the approximating equation (4.2). The functional $V$ is continuous and moreover (4.5) shows that it decreases along the trajectories, thus it remains to show that the third property in the definition of Lyapunov function holds. More precisely, we have to prove that if $V\left(\xi^{N}(t)\right)$ is constant for some complete orbit $\xi^{N}$ then $\xi^{N}$ is a rest point. Now, $z=\left(u^{N}, v^{N}\right)$ is a rest point for $\mathcal{E}_{N}$ if and only if $v^{n}=0$ and $u^{n} \in D\left(A^{1 / 2}\right)$ solves

$$
A u^{n}+\phi\left(u^{n}\right)=0, \text { in } D\left(A^{-1 / 2}\right) .
$$

Thus, to conclude one has to show that $\xi^{N}=\left(u^{N}, 0\right)$ with $u^{N}$ solution of (4.1). This is trivial; in fact if $V\left(\xi^{N}(t)\right)$ is constant for $\xi^{N}=\left(u^{N}, u_{t}^{N}\right)$ then by (4.5) $u_{t}^{N} \equiv 0$ for all $t$ and moreover $u^{N}$ solves (4.24). Thus $\xi$ is a rest point.

Now, $V$ is a Lyapunov functional. Thus, to prove the point dissipativity of $\mathcal{E}_{N}$ we only need to prove that the set of the rest point $Z\left(\mathcal{E}_{N}\right)$ is bounded in the phase space $V^{N} \times V^{N}$ (see [3, Theorem 5.1]). In the following lemma, 
we will show that the set of rest point actually enjoys this boundedness uniformly with respect to $N$. This property will be crucial for the analysis in Section 6.

Lemma 4.1. The set $Z\left(\mathcal{E}_{N}\right)$ of the rest points of (4.2) is bounded in $V^{N} \times$ $V^{N}$ uniformly with respect to $N$. More precisely, there exists $r_{0}$ such that $\forall N \geq 1$ there holds

$$
Z\left(\mathcal{E}_{N}\right) \subset B_{\mathscr{H}}\left(r_{0}\right) .
$$

Proof. If $z^{N}=\left(u^{N}, 0\right)$ is a rest point, then by (4.24) tested with $u^{N}$ we have (recall $(2.3))$

$$
\begin{aligned}
0=\left\|\nabla u^{N}\right\|^{2}+\left\langle u^{N}, \phi\left(u^{n}\right)\right\rangle & \geq\left\|\nabla u^{N}\right\|^{2}-\lambda\left\|u^{N}\right\|^{2}-C \\
& \geq\left(1-\frac{\lambda}{\lambda_{1}}\right)\left\|\nabla u^{N}\right\|^{2}-C,
\end{aligned}
$$

where $\lambda<\lambda_{1}$ and $C$ are constants independent of $u^{N}$ and of $N$. This proves that the set $Z\left(\mathcal{E}_{N}\right)$ of the rest points of $(4.2)$ is bounded in $V^{N} \times V^{N}$ uniformly with respect to $N$.

We are now in the position to state and prove the main result of this section.

Theorem 4.2. For any $N \in \mathbb{N}$ fixed, under the assumptions (2.3) and (2.4), the set of all the approximate solution $\mathcal{E}_{N}$ is a generalized semiflow on the phase space $V^{N} \times V^{N}$. Moreover, it is asymptotically compact and point dissipative, thus it possesses a unique global attractor $\mathscr{A}_{N}$, with the structure (3.4), which attracts bounded sets of $V^{N} \times V^{N}$. Moreover, for each complete orbit $\psi^{N}$ lying in $\mathscr{A}_{N}$ the limit sets $\alpha(\psi)$ and $\omega(\psi)$ are connected subsets of $Z\left(\mathcal{E}_{N}\right)$ on which $V$ is constant. Finally, there exists $R_{0}>0$ such that, for any $N \geq 1$ there holds

$$
\mathscr{A}_{N} \subset B_{\mathscr{H}}\left(R_{0}\right) \cap\left(V^{N} \times V^{N}\right) .
$$

Proof. The proof of the existence and uniqueness of the attractor $\mathscr{A}_{N}$ is an easy consequence of the previous discussion and of Ball's Theorem [3, Theorem 5.1] (but see also Theorem 3.3 in this paper). In particular, in this section we have shown that $\mathcal{E}_{N}$ is a generalized semiflow on $V^{N} \times V^{N}$ which is point dissipative and asymptotically compact. Thus, [3, Theorem 5.1] applies and we deduce the existence of a unique compact attractor $\mathscr{A}_{N}$ for the solutions of (4.2). The property (3.4) comes from Theorem 3.6, which clearly holds also for generalized semiflows.

To conclude, it remains to prove (4.26). Now, we recall from Lemma 4.1 that there exists $r_{0}$ such that

$$
Z\left(\mathcal{E}_{N}\right) \subset B_{\mathscr{H}}\left(r_{0}\right), \text { for any } N \geq 1 \text {. }
$$

Thus, since for any given element $\left(u^{N}, u_{t}^{N}\right) \in \mathcal{E}_{N}$ we have that $\omega\left(u^{N}, u_{t}^{N}\right) \subset$ $Z\left(\mathcal{E}_{N}\right)$, we deduce that setting $r_{1}=2 r_{0}$, we have that the ball $B \mathscr{H}\left(r_{1}\right) \cap$ $\left(V^{N} \times V^{n}\right)$ is an attracting set in $V^{N} \times V^{N}$, i.e. there exists $t_{N}$ such that for any $t \geq t_{N},\left(u^{N}, u_{t}^{N}\right) \in B_{\mathscr{H}}\left(r_{1}\right) \cap\left(V^{N} \times V^{n}\right)$. Now, we consider the positive orbit of $B_{\mathscr{H}}\left(r_{1}\right) \cap\left(V^{N} \times V^{n}\right)$, i.e. the set $C_{1}=\bigcup_{t \geq 0} T_{N}(t)\left(B_{\mathscr{H}}\left(r_{1}\right) \cap\left(V^{N} \times\right.\right.$ $\left.V^{n}\right)$ ), where $T_{N}(t)$ is the multivalued semigroup constructed from $\mathcal{E}_{N}$. Note 
that $C_{1}$ is still an absorbing set which attracts all compact sets of $V^{N} \times V^{N}$ and which is included, thanks to (4.5) and (4.8), in $B_{\mathscr{H}}\left(C\left(r_{1}\right)\right) \cap\left(V^{N} \times V^{n}\right)$, where $C\left(r_{1}\right)$ is as in (4.9). Now, we let the system evolve from $C_{1}$. It is now clear that setting $R_{0}:=C\left(C\left(r_{1}\right)\right)$ we have that

$$
\mathscr{A}^{N}=\omega\left(C_{1}\right) \subset B_{\mathscr{H}}\left(R_{0}\right) \cap\left(V^{N} \times V^{N}\right),
$$

which concludes the proof.

\section{LONG TIME BeHAVIOUR OF THE HYPERBoliC RELAXATION OF THE}

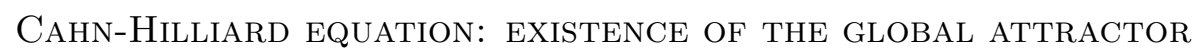

In this section we state and prove our main results concerning the longtime behaviour of (a suitable subset of ) the solutions to (2.5). As already pointed in the Introduction, the class of the solutions to (2.5) for which we study the long-time behaviour will be the set of the functions that solve (2.5) and that are also limit of the approximation argument presented in Section 4 (see Definition 5.1 below). To this end, we first let $u_{0}^{N}$ in (4.3) (resp. $u_{1}^{N}$ ) to be the projection of $u_{0}$ (resp. $\left.u_{1}\right)$ on $V^{N}$. Now, since $u_{0}^{N} \rightarrow u_{0}$ in $D\left(A^{1 / 2}\right)$ and $u_{1}^{N} \rightarrow u_{1}$ in $D\left(A^{-1 / 2}\right)$ by construction, it is clear from (4.5) with $s=0$ that the sequence of approximating solutions $u^{N}$ enjoy the following bound, independently of $N$

$$
\begin{gathered}
\left\|u^{N}\right\|_{L^{\infty}\left(0, T ; D\left(A^{1 / 2}\right)\right)}+\left\|u_{t}^{N}\right\|_{L^{\infty}\left(0, T ; D\left(A^{-1 / 2}\right)\right) \cap L^{2}\left(0, T ; D\left(A^{-1 / 2}\right)\right)} \\
+\left\|F\left(u^{N}\right)\right\|_{L^{\infty}\left(0, T ; L^{1}(\Omega)\right)} \leq C .
\end{gathered}
$$

This means, in particular, that if we are able to pass to the limit as $N \nearrow+\infty$ in (4.2) and find a solution to (2.5), then this solution would satisfy only an energy inequality and no more the energy equality (4.5). In fact, since we are not able to perform further a priori estimates on the approximating solutions, from (5.1) we obtain only the corresponding weak and weak star convergences. This obviously prevents from gaining the energy identity (4.5) in the limit.

This preliminary discussion shows that the correct topology for the phase space $\mathscr{H}:=D\left(A^{1 / 2}\right) \times D\left(A^{-1 / 2}\right)$ should be its weak topology $\sigma\left(\mathscr{H}, \mathscr{H}^{\prime}\right)$. This choice has however a drawback: this weak topology is not metrizable, thus the concepts which are defined using the underlying metric structure of the phase space, should be properly (re)-defined. However, note that the concept of generalized semiflow is of topological nature. This means that we can construct a generalized semiflow when the underlying phase space is just a topological space of Hausdorff type and no more a metric space. On this regard, we borrow an idea from Babin and Vishik [1] (but see also [10]). In particular, we consider as phase space for our equation (2.5) the Hilbert space $\mathscr{H}:=D\left(A^{1 / 2}\right) \times D\left(A^{-1 / 2}\right)$ endowed with the topology induced by the weak convergence. Moreover, we let a metric $\mu$ be defined on $\mathscr{H}$. The metric $\mu$ will be the one given by the norm in $D\left(A^{1 / 2}\right) \times D\left(A^{-1 / 2}\right)$. The only need in introducing this metric $\mu$ is to define the family of bounded sets we will work with. On the contrary, the attracting properties of the global attractor will depend on the weak topology. Consequently, we say that a subset $\mathscr{A}$ of the phase space attracts a set $B$ if there holds

for any $\varepsilon>0$ there exists a $t_{0}>0$ such that $T(t) B \subset N_{\sigma}^{\varepsilon}(A)$ for any $t \geq t_{0}$, 
where $N_{w}^{\varepsilon}(A)$ is an $\varepsilon$-neighbourhood of $A$ in the weak topology.

We refer to [25], [20], for other results in the weak topology of the phase space.

We collect all this informations by saying that the phase space for (2.5) is the couple

$$
\mathcal{X}=(\mathscr{H}, \mu),
$$

where $\mathscr{H}=D\left(A^{1 / 2}\right) \times D\left(A^{-1 / 2}\right)$ with the weak convergence topology

$$
\text { and } \mu\left(\left(u_{1}, u_{2}\right),\left(v_{1}, v_{2}\right)\right)=\sqrt{\left\|u_{1}-v_{1}\right\|_{D\left(A^{1 / 2}\right)}^{2}+\left\|u_{2}-v_{2}\right\|_{D\left(A^{-1 / 2}\right)}^{2}} \text {. }
$$

In particular, we say that a sequence $v_{n}$ converges to $v$ in $\mathcal{X}\left(v_{n} \stackrel{\mathcal{X}}{\rightarrow} v\right)$ when

$$
v_{n} \rightarrow v \text { in } D\left(A^{1 / 2}\right) \times D\left(A^{-1 / 2}\right) .
$$

Now, we are ready to introduce the set of the solutions to (2.5) for which we are going to study the long-time behaviour.

Definition 5.1 (Limiting solutions). We say that a function $\varphi=\left(\begin{array}{c}u \\ u_{t}\end{array}\right) \in$ $L^{\infty}\left(0,+\infty ; D\left(A^{1 / 2}\right) \times D\left(A^{-1 / 2}\right)\right)$ is a limiting solution of (2.5) if $u$ solves the equation (2.5) a.e. on $(0,+\infty)$ and there exists a sequence $\left\{N_{k}\right\}$, and a sequence $\varphi^{N_{k}}=\left(\begin{array}{l}u^{N_{k}} \\ u_{t}^{N_{k}}\end{array}\right) \in \mathcal{E}_{N_{k}}$ for all $k$ such that

$$
\varphi^{N_{k}} \stackrel{\mathcal{X}}{\rightarrow} \varphi \quad \text { in } \mathcal{X} \quad \text { locally uniformly on }[0,+\infty) \text {. }
$$

The first result concerning the set of the limiting solutions is the following

Theorem 5.2. Assume (2.3) and (2.4), then $\overline{\mathcal{E}}$ is a weak generalized semiflow (see Definition 3.4) in $\mathcal{X}$ (see 5.2). Moreover, $\overline{\mathcal{E}}$ complies with $(C 3),(C 4)$ in $\mathcal{X}$.

Proof. First of all, let us notice that as soon as $\overline{\mathcal{E}}$ is non empty, then it satisfies $(H 2)$, as one can readily check. We start with proof of $(H 1)$, which clearly entails a global existence result for (2.5). First of all, (4.4) integrated in time gives a bound for the approximate solutions in the energy space $L^{\infty}(0, T ; \mathscr{H}), \forall T>0$. Thus, since $D\left(A^{1 / 2}\right)$ is continuously embedded in $L^{6}(\Omega)$ in 3 -D, we have that $\phi\left(u^{N}\right)$ is bounded in $L^{\infty}\left(0, T ; L^{2}(\Omega)\right)$ $\forall T>0$ thanks to (2.4). Finally, by reasoning as in (4.15)-(4.16) we obtain that $u_{t t}^{N}$ lies (uniformly with respect to $N$ ) in a bounded subset of $L^{\infty}\left(0, T ; D\left(A^{-3 / 2}\right)\right), \forall T>0$. Now, usual weak star compactness tools entail that there exists a function

$$
\begin{gathered}
u \text { with }\left(u, u_{t}\right) \in L^{\infty}(0, T ; \mathscr{H}) \\
\text { and } u_{t t} \in L^{\infty}\left(0, T ; D\left(A^{-3 / 2}\right)\right) \text { for any } T>0
\end{gathered}
$$

and a subsequence $N_{k}$ of $N$, such that, for any $T>0$,

$$
\begin{gathered}
u_{k}^{N} \stackrel{*}{\rightarrow} u \text { in } L^{\infty}\left(0, T ; D\left(A^{1 / 2}\right)\right), \\
u_{t}^{N_{k}} \stackrel{*}{\rightarrow} u_{t} \text { in } L^{\infty}\left(0, T ; D\left(A^{-1 / 2}\right)\right), \\
u_{t t}^{N_{k}} \stackrel{*}{\rightarrow} u_{t t} \text { in } L^{\infty}\left(0, T ; D\left(A^{-3 / 2}\right)\right),
\end{gathered}
$$


Convergences (5.4)-(5.6) allow to obtain in the limit the first three linear terms in equation (2.5). Concerning the term with $\phi\left(u^{N}\right)$, we follow Ball [4, Lemma 3.3]. Note that, as a consequence of this limit procedure, by the semicontinuity of norms with respect to the weak convergence, the limiting solutions satisfy the energy inequality

$$
\begin{gathered}
V\left(u, u_{t}\right)(t)+\int_{s}^{t}\left\|u_{t}(\lambda)\right\|_{D\left(A^{-1 / 2}\right)}^{2} d \lambda \leq V\left(u, u_{t}\right)(s), \\
\forall t>s, \text { for a.e.s and for } s=0 .
\end{gathered}
$$

Now, by possibly modifying $u$ on a set of times of measure zero, we have that $u:[0,+\infty) \rightarrow D\left(A^{-1 / 2}\right)$ is strongly continuous with $u(0)=u_{0}$ (see [28, Chapter 1, Theorem 3.1]). Hence, since $u \in L^{\infty}\left(0,+\infty ; D\left(A^{1 / 2}\right)\right)$, we easily get that $u:[0,+\infty) \rightarrow D\left(A^{1 / 2}\right)$ is weakly continuous. An analogous argument shows the weak continuity of $u:[0,+\infty) \rightarrow D\left(A^{-1 / 2}\right)$. Thus, $(C 3)$ is fulfilled. To conclude the proof of $(H 1)$, we only need to check the convergence (5.3). Now, for any $t$ we fix a sequence of times $t_{N}$ such that $t_{N} \rightarrow t$. Then, since

$$
u^{N}\left(t_{N}\right)=u_{0}^{N}+\int_{0}^{t_{N}} u_{t}^{N}(s) d s, \quad \text { in } D\left(A^{-1 / 2}\right),
$$

and $u_{t}^{N} \stackrel{*}{\rightarrow} u_{t}$ in $L^{\infty}\left(0, T ; D\left(A^{-1 / 2}\right)\right)$ for any $T>0$, we have that (recall that $u_{0}^{N} \rightarrow u_{0}$ in $\left.D\left(A^{1 / 2}\right)\right)$

$$
\begin{gathered}
\left\langle A^{-1 / 2} u^{N}\left(t_{N}\right), A^{1 / 2} v\right\rangle \rightarrow\left\langle A^{-1 / 2} u_{0}, A^{1 / 2} v\right\rangle+\int_{0}^{t}\left\langle A^{-1 / 2} u_{t}(s), A^{1 / 2} v\right\rangle d s \\
=\left\langle A^{-1 / 2} u(t), A^{1 / 2} v\right\rangle, \quad \forall v \in D\left(A^{1 / 2}\right)
\end{gathered}
$$

which implies that $u^{N}\left(t_{N}\right) \rightarrow u(t)$ in $L^{2}(\Omega)$, thus by density in $D\left(A^{1 / 2}\right)$. Hence, we have that $u^{N} \rightarrow u$ in $D\left(A^{1 / 2}\right)$ locally uniformly in $[0, \infty)$ (recall that $u$ enjoys $(C 3)$ ). A similar argument also shows that $u_{t}^{N} \rightarrow u_{t}$ in $D\left(A^{-1 / 2}\right)$ locally uniformly in $[0, \infty)$. This shows (5.3). Hence, the proof of $(H 1)$ is concluded.

We now prove $(C 4)$, which obviously yields (H4). Let us fix a sequence $\varphi_{m}=\left(\begin{array}{c}u_{m} \\ u_{m t}\end{array}\right) \subset \overline{\mathcal{E}}$ with $\varphi_{m}(0) \stackrel{\mathcal{X}}{\rightarrow} z=\left(\begin{array}{l}u_{0} \\ u_{1}\end{array}\right)$. We aim to show that there exists $\varphi=\left(\begin{array}{c}u \\ u_{t}\end{array}\right) \in \overline{\mathcal{E}}$ such that, up to a subsequence, $\varphi_{m} \stackrel{\mathcal{X}}{\rightarrow} \varphi$ locally uniformly in $[0, \infty)$. By definition of $\overline{\mathcal{E}}$, for all $m$ there exists a sequence $\left\{\varphi_{m}^{N_{k}}\right\} \subset \mathcal{E}_{N_{k}}$ such that $\varphi_{m}^{N_{k}} \stackrel{\mathcal{X}}{\rightarrow} \varphi_{m}$ locally uniformly on $[0, \infty)$. This fact entails that we can select a proper increasing sequence $N_{k_{m}}$ (named for simplicity $N_{m}$ ) such that

$$
\sup _{t \in[0, m]}\left\langle\left\langle\varphi_{m}(t)-\varphi_{m}^{N_{m}}(t), v\right\rangle\right\rangle \leq \frac{1}{m}, \quad \forall v \in \mathscr{H}^{*} .
$$

This means in particular that $\varphi_{m}^{N_{m}}(0) \stackrel{\mathcal{X}}{\rightarrow} z$. Now, thanks to (4.4) and standard diagonal weak compactness arguments (analogous to the ones used 
in proving $(H 1)$ ), we have (up to a subsequence)

$$
\begin{aligned}
N_{m_{j}}^{N_{m_{j}}} \stackrel{*}{\rightarrow} u \text { in } L^{\infty}\left(0, T ; D\left(A^{1 / 2}\right)\right), & \forall T>0 \\
u_{m_{j} t}^{N_{m_{j}}} \stackrel{*}{\rightarrow} u_{t} \text { in } L^{\infty}\left(0, T ; D\left(A^{-1 / 2}\right)\right) & \forall T>0 \\
u_{m_{j} t t}^{N_{m_{j}}} \stackrel{*}{\rightarrow} u_{t t} \text { in } L^{\infty}\left(0, T ; D\left(A^{-3 / 2}\right)\right) & \forall T>0 \\
\phi\left(u_{m_{j}}^{N_{m_{j}}} \stackrel{*}{\rightarrow} \phi(u) \text { in } L^{\infty}\left(0, T ; L^{2}(\Omega)\right)\right. & \forall T>0,
\end{aligned}
$$

as $j \nearrow+\infty$. Thus, $u$ solves (2.5). Moreover, we have also (repeating exactly the same argument we use in proving (5.3) that

$$
\begin{gathered}
u_{m_{j}}^{N_{m_{j}}} \rightarrow u \text { in } D\left(A^{1 / 2}\right) \text { locally uniformly in }[0, \infty) \\
u_{m_{j} t}^{N_{m_{j}}} \rightarrow u_{t} \text { in } D\left(A^{-1 / 2}\right) \text { locally uniformly in }[0, \infty)
\end{gathered}
$$

This means in particular that $\varphi:=\left(\begin{array}{c}u \\ u_{t}\end{array}\right)$ belongs to $\overline{\mathcal{E}}$. Now, we conclude the proof of $(C 4)$ by noting that, for all $T$ and $m_{j}>T$ there holds (see $(5.10))$

$$
\begin{gathered}
\sup _{t \in[0, T]}\left\langle\left\langle\varphi(t)-\varphi_{m_{j}}(t), v\right\rangle\right\rangle \\
\leq \sup _{t \in[0, T]}\left\langle\left\langle\varphi(t)-\varphi_{m_{j}}^{N_{m_{j}}}(t), v\right\rangle\right\rangle+\sup _{t \in[0, T]}\left\langle\left\langle\varphi_{m_{j}}^{N_{m_{j}}}-\varphi_{m_{j}}(t), v\right\rangle\right\rangle \\
\leq \sup _{t \in[0, T]}\left\langle\left\langle\varphi(t)-\varphi_{m_{j}}^{N_{m_{j}}}(t), v\right\rangle\right\rangle+\frac{1}{m_{j}}, \quad \forall v \in \mathscr{H}^{*} .
\end{gathered}
$$

By recalling (5.15-5.16), we have that (5.17) clearly entails the validity of $(C 4)$ in $\mathcal{X}$.

We now prove that $\overline{\mathcal{E}}$ is asymptotically compact and point dissipative. On this regard, we note that now the functional $V$ is not a Lyapunov function for $\overline{\mathcal{E}}$, since it is only lower semicontinuous in $\mathcal{X}$. Thus, the study of the dissipation properties of $\overline{\mathcal{E}}$ is quite different form the one for $\mathcal{E}_{N}$. In particular, it is no more true that any neighbourhood of the set of rest points is an absorbing set for $\overline{\mathcal{E}}$. On the other hand, trivial counterexamples show that we can not deduce the dissipativity of the limiting solution from the dissipativity of their approximations. Consequently, we will make use of a proper (although quite standard) dissipativity estimate. To this end, we have to reinforce the conditions on $\phi$ (see (5.18) below). However, note that the forthcoming assumption (5.18), which basically amounts to saying that $r \phi(r)$ is controlled for large $r$ from above and from below by a proper power of $|r|$, is quite standard and widely used (see for instance [10]). Moreover, it is satisfied by the cubic nonlinearity $\phi(r)=r^{3}-r$.

Theorem 5.3. Under the hypotheses of Theorem 5.2, the set $\overline{\mathcal{E}}$ is asymptotically compact in $\mathcal{X}$ (see (5.2)). Moreover, if there holds the following 
condition

$$
\liminf _{|r| \nearrow+\infty} \frac{r \phi(r)-c F(r)}{|r|^{2}} \geq 0
$$

for some $c>0$, then there exists $\bar{R}>0$ such that the ball $B_{\mathcal{X}}(\bar{R})$ of $\mathcal{X}$ is a bounded absorbing set for $\overline{\mathcal{E}}$. Thus, the set $\overline{\mathcal{E}}$ is point dissipative.

Proof. The asymptotic compactness easily follows from the energy estimate (4.4) integrated between 0 and $t_{m}$ with $t_{m} \nearrow+\infty$. In fact, given a sequence $\varphi_{m}=\left(\begin{array}{c}u_{m} \\ u_{m t}\end{array}\right) \subset \overline{\mathcal{E}}$ with $\varphi_{m}(0)$ bounded in $\mathcal{X}$, then the corresponding solutions evaluated at $t_{m}$ enjoy

$$
V\left(u_{m}\left(t_{m}\right), u_{m t}\left(t_{m}\right)\right)+\int_{0}^{t_{m}}\left\|u_{m t}(s)\right\|_{D\left(A^{-1 / 2}\right)} d s \leq C .
$$

This clearly implies that, up to a subsequence, $\varphi_{m}\left(t_{m}\right)=\left(\begin{array}{c}u_{m}\left(t_{m}\right) \\ u_{m t}\left(t_{m}\right)\end{array}\right)$ is convergent in $\mathcal{X}$.

We now prove the point dissipativity of $\overline{\mathcal{E}}$. To this end, we test (2.5) with $A^{-1} u_{t}+\alpha A^{-1} u^{1}$ with $\alpha>0$ small enough. We set

$$
\Phi\left(u, u_{t}\right):=\frac{1}{2}\left\|u_{t}\right\|_{D\left(A^{-1 / 2}\right)}^{2}+\frac{1}{2}\|\nabla u\|^{2}+\int_{\Omega} F(u) d x+\alpha\left\langle A^{-1 / 2} u_{t}, A^{-1 / 2} u\right\rangle,
$$

and we get

$$
\begin{gathered}
\frac{d}{d t} \Phi\left(u, u_{t}\right)+(1-\alpha)\left\|u_{t}\right\|^{2}+\alpha\|\nabla u\|^{2} \\
+\alpha\left\langle A^{-1 / 2} u_{t}, A^{-1 / 2} u\right\rangle+\alpha\langle\phi(u), u\rangle=0 .
\end{gathered}
$$

Note that, by choosing $\alpha$ small enough, from (2.3), (4.7), the Poincaré and the Young inequalities, it is not difficult to obtain that $I$ is bounded from below by

$$
\Phi\left(u, u_{t}\right) \geq c\left(\left\|u_{t}\right\|_{D\left(A^{-1 / 2}\right)}^{2}+\|\nabla u\|^{2}\right) .
$$

Now, by adding and subtracting $\int_{\Omega} F(u) d x$ in (5.20), we get

$$
\frac{d}{d t} \Phi\left(u, u_{t}\right)+\Phi\left(u, u_{t}\right)+\alpha\langle\phi(u), u\rangle-\int_{\Omega} F(u) d x=0 .
$$

To control the last term in (5.22), we use (5.18), which entails that, for any $\varepsilon>0$ the exists a constant $C_{\varepsilon}$ such that

$$
\alpha\langle u, \phi(u)\rangle-\int_{\Omega} F(u)+\varepsilon\|u\|^{2} \geq C_{\varepsilon} .
$$

Thus, by choosing $\varepsilon$ in (5.23) small enough, (5.22) becomes

$$
\frac{d}{d t} I\left(u, u_{t}\right)+I\left(u, u_{t}\right) \leq C .
$$

The Gronwall Lemma applies and we deduce the fundamental

$$
I\left(u(t), u_{t}(t)\right) \leq C I\left(u(0), u_{t}(0)\right) e^{-t}+C,
$$

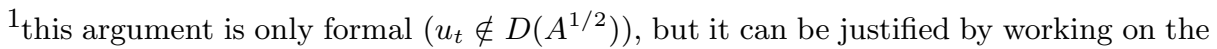
Faedo-Galerkin scheme and recalling the definition of $\overline{\mathcal{E}}$.
} 
which, by recalling (5.21), clearly entails the desired dissipativity.

We are now in the position to state the main result of this section, which is now an easy consequence of Theorems 5.2 and 5.3 and of Theorem 3.6.

Theorem 5.4. Assume (2.3), (2.4) and (5.18). Then the set $\overline{\mathcal{E}}$ of all the limiting solutions to (2.5) (see Definition 5.1) possesses a weak global attractor $\mathscr{A}$ in $\mathscr{H}$ endowed with the weak convergence. The attractor $\mathscr{A}$ attracts in the weak topology of $\mathscr{H}$ the $\mathscr{H}$-norm bounded subsets of $\mathscr{H}$. Moreover, $\mathscr{A}$ enjoys the description (3.4).

Proof. From Theorem 5.2 we learn that the set $\overline{\mathcal{E}}$ of all the limiting solutions to $(2.5)$ enjoys $(H 1),(H 2),(H 4)$ and $(C 4)$ in $\mathcal{X}$ (see (5.2)). From Theorem 5.3 , we know that $\overline{\mathcal{E}}$ is asymptotically compact and point dissipative. Thus, Theorem 3.6 applies in the phase space $\mathcal{X}$.

\section{Approximation of the Weak global attractor}

In this section we discuss about the approximability of the (weak) global attractor $\mathscr{A}$ for $(2.5)$ with the global attractor $\mathscr{A}_{N}$ of the Faedo-Galerkin system (4.2). To this end we first introduce some notation. Given a subset $B \subset \mathcal{X}$ and any $\varepsilon>0$, we let $O_{w}^{\varepsilon}(B)$ to denote the open $\varepsilon$-neighbourhood of $B$ in the weak topology, that is the topology related to the phase space (5.2).

This is the main result of this section.

Theorem 6.1. Let (2.3)-(2.4) hold. Then, for any $\varepsilon>0$, there exists $N_{\varepsilon}$ such that $\mathscr{A}_{N} \subseteq O_{w}^{\varepsilon}(\mathscr{A})$ for any $N \geq N_{\varepsilon}$.

Proof. To prove this result, we combine the result (3.4) on the structure of the weak global attractor $\mathscr{A}$ and of $\mathscr{A}^{N}$ with the uniform (in $N$ ) boundedness of $\mathscr{A}_{N}$ in $\mathcal{X}$ (see (4.26) and (5.2)).

In order to prove the Theorem, we argue by contradiction. Thus, there exists an open $\bar{\varepsilon}$-neighbourhood of $\mathscr{A} O_{w}^{\bar{\varepsilon}}(\mathscr{A})$ such that

$$
\boldsymbol{\xi}^{N_{j}}=\left(\xi_{1}^{N_{j}}, \xi_{2}^{N_{j}}\right) \notin O_{w}^{\bar{\varepsilon}}(\mathscr{A})
$$

for a sequence $N_{j} \nearrow+\infty$ and $\boldsymbol{\xi}^{N_{j}} \in \mathscr{A}^{N_{j}}$. Now, thanks to (4.26), we have that

$$
\left\|\boldsymbol{\xi}^{N_{j}}\right\|_{\mathscr{H}} \leq R_{0},
$$

which clearly means that, up to the extraction of a (not relabeled subsequence),

$$
\boldsymbol{\xi}^{N_{j}} \stackrel{\mathcal{X}}{\rightarrow} \boldsymbol{\xi}
$$

Our goal is to prove that $\boldsymbol{\xi}$ belongs to $\mathscr{A}$, hence obtaining a contradiction. To this end, we note that the quasi invariance of the global attractor $\mathscr{A}_{N}$ gives that there exists a complete and bounded orbit $\varphi^{N}=\left(u^{N_{j}}, u_{t}^{N_{j}}\right)$ such that $\varphi^{N_{j}}(0)=\boldsymbol{\xi}^{N_{j}}$ and $\varphi^{N}(t) \in \mathscr{A}_{N}$ for all $t \in \mathbb{R}$. As a direct consequence of the translation invariance of the complete orbit, of (6.1), (4.5), (4.8) and by reasoning as in the proof of Theorem 5.2, we have that the complete orbit $\varphi^{N_{j}}$ satisfies, for any $T>0$, the following

$$
\left\|u_{t t}^{N_{j}}\right\|_{L^{\infty}\left(-T, T ; D\left(A^{-3 / 2}\right)\right)}+\left\|\left(u^{N_{j}}, u_{t}^{N_{j}}\right)\right\|_{L^{\infty}(-T, T ; \mathscr{H})} \leq C\left(R_{0}\right) .
$$


As already pointed out in the proof of Theorem 5.2, this estimate guarantees the existence of a limiting solution $\varphi$, obtained as the limit (up to the extraction of a further subsequence) of the $\varphi^{N_{j}}$ 's, which is also a bounded and complete orbit. Moreover, there holds that $\varphi(0)=\boldsymbol{\xi}$. From the structure result (3.4) in Theorem 5.4 we finally deduce that $\boldsymbol{\xi} \in \mathscr{A}$. This clearly leads to a contradiction.

\section{WEAK GLOBAL ATtRACTOR FOR THE DAMPED SEMILINEAR WAVE EQUATION WITH SUPERCRITICAL NONLINEARITY}

In this last section of the paper, we show that the same type of results obtained for the hyperbolic relaxation of the Cahn-Hiliard equation hold also for semilinear damped wave equation when the non linear term $\phi$ has a super critical growth and is not locally lipschitz continuous. Thus we will deal with the following equation

$$
u_{t t}+u_{t}-\Delta u+\phi(u)=0 \quad \text { in } \Omega \subset \mathbb{R}^{n}
$$

with $\phi: \mathbb{R} \rightarrow \mathbb{R}$ a continuous function with $\phi(r)=F^{\prime}(r)$ satisfying $(2.3)$ and

\section{Conditions on $\phi$}

$$
\begin{aligned}
&|\phi(v)| \leq C\left(1+|v|^{\gamma}\right), \quad C>0, \quad 1 \leq \gamma<+\infty, \\
& \liminf _{|u| \nearrow+\infty} \frac{\frac{1}{2} u \phi(u)-F(u)}{|u|^{\gamma+1} \geq 0,} \\
& \liminf _{|u| \nearrow+\infty}\left(\frac{\phi(u)}{u}-C|u|^{\gamma+1}\right)>-\lambda_{1}, \quad C>0 \\
& F(u) \geq C|u|^{\gamma+1}-C, \quad C>0 \text { if } \gamma>\frac{n+2}{n-2}
\end{aligned}
$$

This conditions are taken from [4]. Moreover, following Ball's notation, we set $Y_{\gamma}=H_{0}^{1}(\Omega) \cap L^{\gamma+1}(\Omega)$ with dual $Y_{\gamma}^{\prime}=H^{-1}(\Omega)+L^{\frac{\gamma+1}{\gamma}}(\Omega)$. Then, we take $X_{\gamma}=Y_{\gamma} \times L^{2}(\Omega)$.

In Ball's paper [4] it is proved that under the hypothesis (7.2)-(7.5), the weak solutions to (7.1) generates a generalized semiflow on the phase space $X_{\gamma}=Y_{\gamma} \times L^{2}(\Omega)$ possessing a global attractor. The key (but unproved) assumption is that the solutions to (7.1) enjoy the energy identity

$\frac{1}{2}\left\|u_{t}(t)\right\|^{2}+\frac{1}{2}\|\nabla u(t)\|^{2}+\int_{0}^{t}\left\|u_{t}(s)\right\|^{2}=\frac{1}{2}\left\|u_{t}(0)\right\|^{2}+\frac{1}{2}\|\nabla u(0)\|^{2}, \quad t \in(0,+\infty)$.

In [27], the existence of solutions to (7.1) is proved by means of the following Faedo Galerkin approximating scheme. Following Lions, we first introduce a sequence of functions $\left\{v_{i}\right\}_{i \in \mathbb{N}}$ such that

$$
\left\{\begin{array}{l}
v_{i} \in H_{0}^{1}(\Omega) \cap L^{\gamma+1}(\Omega) \forall i \\
\forall N, v_{1}, \ldots, v_{N} \text { are linearly independent } ; \\
\forall N, v_{1}, \ldots, v_{N} \text { spans a dense subset of } H_{0}^{1}(\Omega) \cap L^{\gamma+1}(\Omega) .
\end{array}\right.
$$

The Faedo-Galerkin system reads thus as follows 
$\operatorname{Problem}_{N}$ : Find $t_{N}$ and $u_{i} \in C^{2}\left(\left[0, t_{N}\right]\right), i=1, \ldots, N$ such that, if

$$
u^{n}:=\sum_{i=1}^{N} u_{i}(t) v_{i},
$$

then $u^{N} \in C^{2}\left(\left[0, t_{N}\right], V_{N}\right)$ solves $(i=1, \ldots, N)$

$$
\begin{gathered}
\left\langle u_{t t}^{N}, v_{i}\right\rangle+\left\langle u_{t}^{N}, v_{i}\right\rangle+\left\langle\nabla u^{N}, \nabla v_{i}\right\rangle+\left\langle\phi\left(u^{N}\right), v_{i}\right\rangle=0, \\
\left\langle u^{N}(0)-u_{0}^{N}, v_{i}\right\rangle=0, \quad\left\langle u_{t}^{N}(0)-u_{1}^{N}, v_{i}\right\rangle=0,
\end{gathered}
$$

where $\left(u_{0}^{N}, u_{1}^{N}\right)$ belongs to the discrete space $V^{N} \times V^{N}$, with $V^{N}$ spanned by $\left\{v_{1}, \ldots, v_{N}\right\}$. (7.8)-(7.9) is a system of nonlinear ODE, for which standard methods ensures the existence of at least one local solution defined on $\left[0, t_{N}\right]$. We first show that these solutions are defined over all the positive line. To this end, we multiply (7.8) with $u_{i}^{\prime}$ and then we sum over $i$. We deduce that

$$
\begin{aligned}
& V\left(u^{N}(t), u_{t}^{N}(t)\right)+\int_{s}^{t}\left\|u_{t}^{N}(\lambda)\right\|^{2} d \lambda \\
& =V\left(u^{N}(s), u_{t}^{N}(s)\right), \quad \forall t, s \in[0,+\infty),
\end{aligned}
$$

where we have set

$$
V\left(u^{N}, u_{t}^{N}\right)(t):=\frac{1}{2}\left\|u_{t}^{N}(t)\right\|^{2}+\frac{1}{2}\left\|\nabla u^{N}(t)\right\|^{2}+\int_{\Omega} F\left(u^{N}(t)\right) .
$$

Thus, (7.5) entails that the solutions are globally defined. Moreover, we have that $\forall N$ the solutions of the Faedo Galerkin system verify the energy identity. Now, by simply mimicking the analysis in [4] and the argument in Theorem 4.2 , we deduce that

(1) the solutions of the Faedo Galerkin system form a generalized semiflow $\mathcal{E}_{N}$ on the discrete space $V^{N} \times V^{N}$

(2) $\mathcal{E}_{N}$ is asymptotically compact and point dissipative, thus possessing a global attractor $\mathscr{A}_{N}$ which is bounded in $X_{\gamma}$ (uniformly w.r.t. $N$ ).

Subsequently, we choose $u_{0}^{N} \rightarrow u_{0}$ in $Y_{\gamma}$ and $u_{1}^{N} \rightarrow u_{1}$ in $L^{2}(\Omega)$ and, as we did in (5.2), we choose as phase space for the continuous problem

$$
\mathcal{X}_{\gamma}=\left(X_{\gamma}, \mu\right)
$$

where $X_{\gamma}=Y_{\gamma} \times L^{2}(\Omega)$ with the weak convergence topology and $\mu$ is the distance induced by norm in $X_{\gamma}$.

As in the analysis of equation (2.5), we denote the convergence in $\mathcal{X}_{\gamma}$ with $\stackrel{\mathcal{X}_{\gamma}}{\rightarrow}$, which simply means weak convergence in $X_{\gamma}$. Then, we introduce the set of the limiting solutions to (7.1) as

Definition 7.1 (Limiting solutions). We say that a function $\varphi=\left(\begin{array}{c}u \\ u_{t}\end{array}\right) \in$ $L^{\infty}\left(0,+\infty ; X_{\gamma}\right)$ is a limiting solution of (7.1) if $u$ solves the equation (7.1) a.e. on $(0,+\infty)$ and there exists a sequence $\left\{N_{k}\right\}$, and a sequence $\varphi^{N_{k}}=$ $\left(\begin{array}{l}u^{N_{k}} \\ u_{t}^{N_{k}}\end{array}\right) \in \mathcal{E}_{N_{k}}$ for all $k$ such that

$$
\varphi^{N_{k}} \stackrel{\mathcal{X}_{\gamma}}{\rightarrow} \varphi \quad \text { in } \mathcal{X}_{\gamma} \quad \text { locally uniformly on }[0,+\infty)
$$


Finally, by simply adapting the proofs of Theorems 5.2, 5.3, 5.4 and 6.1, we can show that

(1) the set of the limiting solutions (see the definition below) forms a weak generalized semiflow on the phase space $\mathcal{X}_{\gamma}$ which possesses a weak global attractor $\mathscr{A}$

(2) the convergence of the global attractor $\mathscr{A}_{N}$ to the weak global attractor $\mathscr{A}$, that is $\forall \varepsilon>0, \exists N_{\varepsilon}: \mathscr{A}_{n} \subseteq O_{w}^{\varepsilon}(\mathscr{A}), \forall N \geq N_{\varepsilon}$, where $O_{w}^{\varepsilon}(\mathscr{A})$ denotes the $\varepsilon$-neighbourhood of $\mathscr{A}$ in the weak topology of $X_{\gamma}$.

Note that these results hold without assuming that the solutions enjoy the energy identity (7.6). On the other hand, our results hold in the weak topology of the phase space $X_{\gamma}$ and the attractor we construct is only quasi invariant and no more invariant. We refer to, e.g., [1] and to [9], for other results on the long time behaviour of the damped semilinear wave equation without uniqueness. In particular, the work [1] makes use of the multivalued semigroup method, while in the work [9] the authors use the trajectory method

Acknowledgements I am grateful to Maurizio Grasselli for having proposed me this subject and for many inspiring conversations.

\section{REFERENCES}

[1] A. V. Babin and M. I. Vishik. Maximal attractors of semigroups corresponding to evolutionary differential equations. Mat. Sb. (N.S.), 126(168)(3):397-419, 432, 1985.

[2] J. M. Ball. Measurability and continuity conditions for nonlinear evolutionary processes. Proc. Amer. Math. Soc., 55(2):353-358, 1976.

[3] J. M. Ball. Continuity properties and global attractors of generalized semiflows and the Navier-Stokes equations. J. Nonlinear Sci., 7(5):475-502, 1997.

[4] J. M. Ball. Global attractors for damped semilinear wave equations. Discrete Contin. Dyn. Syst., 10(1-2):31-52, 2004. Partial differential equations and applications.

[5] P. W. Bates and J. Han. The Neumann boundary problem for a nonlocal CahnHilliard equation. J. Differential Equations, 212(2):235-277, 2005.

[6] A. Bonfoh. Existence and continuity of uniform exponential attractors for a singular perturbation of a generalized Cahn-Hilliard equation. Asymptot. Anal., 43(3):233247, 2005.

[7] J.W. Cahn. On spinodal decomposition. Acta Metall, 9:795-801, 1961.

[8] J.W. Cahn and Hilliard J.E. Free energy of a nonuniform system. i. interfacial free energy. J. Chem. Phys., 28:258-267, 1958.

[9] V. V. Chepyzhov and M. I. Vishik. Global attractor and its perturbations for a dissipative hyperbolic equation. Russ. J. Math. Phys., 8(3):251-266, 2001.

[10] V. V. Chepyzhov and M. I. Vishik. Attractors for equations of mathematical physics, volume 49 of American Mathematical Society Colloquium Publications. American Mathematical Society, Providence, RI, 2002.

[11] P. Colli, P. Krejci, E. Rocca, and J. Sprekels. Nonlinear evolution inclusion arising from phase change models. preprint IMATI (CNR), PV, (24):1-28, 2004.

[12] A. Debussche. A singular perturbation of the Cahn-Hilliard equation. Asymptotic Anal., 4(2):161-185, 1991.

[13] M. Efendiev, A. Miranville, and S. Zelik. Exponential attractors for a singularly perturbed Cahn-Hilliard system. Math. Nachr., 272:11-31, 2004. 
[14] E. Feireisl. Global attractors for semilinear damped wave equations with supercritical exponent. J. Differential Equations, 116(2):431-447, 1995.

[15] P. C. Fife. Models for phase separation and their mathematics. Electron. J. Differential Equations, pages No. 48, 26 pp. (electronic), 2000.

[16] H. Gajewski and K. Zacharias. On a nonlocal phase separation model. J. Math. Anal. Appl., 286(1):11-31, 2003.

[17] P. Galenko. Phase-field models with relaxation of the diffusion flux in nonequilibrium solidification of a binary system. Phys. Lett. A, 287(3-4):190-197, 2001.

[18] S. Gatti, M. Grasselli, A. Miranville, and V. Pata. On the hyperbolic relaxation of the one-dimensional Cahn-Hilliard equation. J. Math. Anal. Appl., 312(1):230-247, 2005.

[19] S. Gatti, M. Grasselli, V. Pata, and A. Miranville. Hyperbolic relaxation of the viscous Cahn-Hilliard equation in 3-D. Math. Models Methods Appl. Sci., 15(2):165-198, 2005.

[20] F. Gazzola and M. Sardella. Attractors for families of processes in weak topologies of Banach spaces. Discrete Contin. Dynam. Systems, 4(3):455-466, 1998.

[21] M. E. Gurtin. Generalized Ginzburg-Landau and Cahn-Hilliard equations based on a microforce balance. Phys. D, 92(3-4):178-192, 1996.

[22] J. K. Hale. Asymptotic behaviour and dynamics in infinite dimensions. In Nonlinear differential equations (Granada, 1984), volume 132 of Res. Notes in Math., pages 1-42. Pitman, Boston, MA, 1985.

[23] J. K. Hale, X. Lin, and G. Raugel. Upper semicontinuity of attractors for approximations of semigroups and partial differential equations. Math. Comp., 50(181):89-123, 1988.

[24] J. K. Hale and G. Raugel. Upper semicontinuity of the attractor for a singularly perturbed hyperbolic equation. J. Differential Equations, 73(2):197-214, 1988.

[25] J. K. Hale and N. Stavrakakis. Compact attractors for weak dynamical systems. Appl. Anal., 26(4):271-287, 1988

[26] L. Kapitanski. Minimal compact global attractor for a damped semilinear wave equation. Comm. Partial Differential Equations, 20(7-8):1303-1323, 1995.

[27] J.-L. Lions. Quelques méthodes de résolution des problèmes aux limites non linéaires. Dunod, 1969.

[28] J.-L. Lions and E. Magenes. Non-homogeneous boundary value problems and applications. Vol. I. Springer-Verlag, New York, 1972. Translated from the French by P. Kenneth, Die Grundlehren der mathematischen Wissenschaften, Band 181.

[29] O. Lopes. Compact attractor for a nonlinear wave equation with critical exponent. Proc. Roy. Soc. Edinburgh Sect. A, 115(1-2):61-64, 1990.

[30] S. Maier-Paape and T. Wanner. Spinodal decomposition for the Cahn-Hilliard equation in higher dimensions. I. Probability and wavelength estimate. Comm. Math. Phys., 195(2):435-464, 1998.

[31] S. Maier-Paape and T. Wanner. Spinodal decomposition for the Cahn-Hilliard equation in higher dimensions: nonlinear dynamics. Arch. Ration. Mech. Anal., 151(3):187-219, 2000.

[32] V. S. Melnik and J. Valero. On global attractors of multivalued semiprocesses and nonautonomous evolution inclusions. Set-Valued Anal., 8(4):375-403, 2000

[33] A. Milani and S. Zheng. Exponential attractors and inertial manifolds for singular perturbations of the Cahn-Hilliard equations. Nonlinear Anal., 57(5-6):843-877, 2004.

[34] A. Milani and S. Zheng. Global attractors for singular perturbations of the CahnHilliard equations. J. Differential Equations, 209(1):101-139, 2005.

[35] A. Miranville and S. Zelik. Robust exponential attractors for Cahn-Hilliard type equations with singular potentials. Math. Methods Appl. Sci., 27(5):545-582, 2004.

[36] A. Miranville and S. Zelik. Exponential attractors for the Cahn-Hilliard equation with dynamic boundary conditions. Math. Methods Appl. Sci., 28(6):709-735, 2005.

[37] I. Moise, R. Rosa, and X. Wang. Attractors for noncompact nonautonomous systems via energy equations. Discrete Contin. Dyn. Syst., 10(1-2):473-496, 2004. Partial differential equations and applications.

[38] A. Novick-Cohen. The Cahn-Hilliard equation: mathematical and modeling perspectives. Adv. Math. Sci. Appl., 8(2):965-985, 1998. 
[39] A. Ould Elmounir and F. Simondon. Attracteurs compacts pour des problèmes d'évolution sans unicité. Ann. Fac. Sci. Toulouse Math. (6), 9(4):631-654, 2000.

[40] R. Rossi, A. Segatti, and U. Stefanelli. Attractors for gradient flows of non convex functionals and applications. in preparation, 2005.

[41] G. R. Sell and Y. You. Dynamics of evolutionary equations, volume 143 of Applied Mathematical Sciences. Springer-Verlag, New York, 2002.

[42] R. Temam. Infinite-dimensional dynamical systems in mechanics and physics, volume 68 of Applied Mathematical Sciences. Springer-Verlag, New York, second edition, 1997.

Diparimento di Matematica "F. Casorati", Universitá di Pavia, Via Ferrata 1, 27100 PAVIA, ITALY

E-mail address: antonio.segatti@unipv.it 